\title{
A nonunitary interpretation for a single vector leptoquark combined explanation to the $B$-decay anomalies
}

\section{Hati, J. Kriewald, J. Orloff and A.M. Teixeira}

Laboratoire de Physique de Clermont (UMR 6533), CNRS/IN2P3, Univ. Clermont Auvergne, 4 Av. Blaise Pascal, F-63178 Aubière Cedex, France

E-mail: chandan.hati@clermont.in2p3.fr,

jonathan.kriewald@clermont.in2p3.fr, jean.orloff@clermont.in2p3.fr, ana.teixeira@clermont.in2p3.fr

ABSTRACT: In order to simultaneously account for both $R_{D^{(*)}}$ and $R_{K^{(*)}}$ anomalies in $B$-decays, we consider an extension of the Standard Model by a single vector leptoquark field, and study how one can achieve the required lepton flavour non-universality, starting from a priori universal gauge couplings. While the unitary quark-lepton mixing induced by $\mathrm{SU}(2)_{L}$ breaking is insufficient, we find that effectively nonunitary mixings hold the key to simultaneously address the $R_{K^{(*)}}$ and $R_{D^{(*)}}$ anomalies. As an intermediate step towards various UV-complete models, we show that the mixings of charged leptons with additional vector-like heavy leptons successfully provide a nonunitary framework to explain $R_{K^{(*)}}$ and $R_{D^{(*)}}$. These realisations have a strong impact for electroweak precision observables and for flavour violating ones: isosinglet heavy lepton realisations are already excluded due to excessive contributions to lepton flavour violating $Z$-decays. Furthermore, in the near future, the expected progress in the sensitivity of charged lepton flavour violation experiments should allow to fully probe this class of vector leptoquark models.

Keywords: Beyond Standard Model, Heavy Quark Physics

ARXIV EPRINT: 1907.05511 


\section{Contents}

1 Introduction 2

2 Towards a nonunitarity interpretation of vector leptoquark couplings $\quad 4$

2.1 Accounting for $R_{K^{(*)}}$ and $R_{D^{(*)}}$ in a minimal $V_{1}$ leptoquark framework 5

2.2 Vector-like fermions and "effective" nonunitary mixings in the light sector 5

3 Explaining LFUV data with nonunitary couplings: phenomenological viability

3.1 New contributions to $R_{K^{(*)}}$ and $R_{D^{(*)}}$

3.2 Constraints from (rare) flavour processes, EW precision observables and direct searches

3.3 Results and discussion

4 Concluding remarks

A Constraints from flavour violating rare meson decays and neutral meson mixing

A.1 Exclusive $d_{j} \rightarrow d_{i} \ell^{-} \ell^{\prime+}$ decays 20

A.2 $P \rightarrow \ell^{-} \ell^{\prime+}$ decays 21

A.3 $P \rightarrow P^{\prime} \ell^{-} \ell^{\prime+}$ decays $\quad 22$

A.4 Loop effects in neutrino modes $\quad 23$

A.5 Loop effects in neutral meson mixing 24

B Constraints from cLFV decays $\quad \mathbf{2 5}$

B.1 Radiative lepton decays $\ell_{i} \rightarrow \ell_{j} \gamma \quad 25$

B.2 Three body decays $\ell \rightarrow \ell^{\prime} \ell^{\prime} \ell^{\prime} \quad 26$

B.3 Neutrinoless $\mu-e$ conversion 28

$\begin{array}{lr}\text { C Electroweak precision observables } & \mathbf{2 8}\end{array}$

$\begin{array}{ll}\text { C.1 Couplings of the } Z \text { boson and photon } & 29\end{array}$

C.2 Couplings of the $W$ boson 30

$\begin{array}{lll}\text { C.3 Constraining EWP observables } & 30\end{array}$

$\begin{array}{ll}\text { D Details of the numerical analysis } & 31\end{array}$ 


\section{Introduction}

In the Standard Model (SM), gauge interactions are strictly flavour universal, as confirmed by precision measurements of several electroweak observables, such as $Z \rightarrow \ell \ell$ decays [1, 2]. Recently, a number of observables related to $B$-meson semileptonic decays has started exhibiting slight deviations, from their SM predictions, a.k.a. anomalies, suggesting the possibility of lepton flavour universality violation (LFUV). The most robust LFU-sensitive measurements arise from ratios of individual decay modes, where the theoretical hadronic uncertainties (e.g. from form factors) cancel out, such as the ratio $R_{D^{(*)}}$ between charged current decays, or the ratio $R_{K^{(*)}}$ between neutral current decays, respectively defined as

$$
R_{D^{(*)}}=\frac{\mathrm{BR}\left(B \rightarrow D^{(*)} \tau^{-} \bar{\nu}\right)}{\mathrm{BR}\left(B \rightarrow D^{(*)} \ell^{-} \bar{\nu}\right)}, \quad R_{K^{(*)}}=\frac{\mathrm{BR}\left(B \rightarrow K^{(*)} \mu^{+} \mu^{-}\right)}{\mathrm{BR}\left(B \rightarrow K^{(*)} e^{+} e^{-}\right)},
$$

where $\ell=e, \mu$. Several experiments have reported deviations from the theoretical LFU SM expectations [3-15]. Quantitatively, the current measured values of $R_{D}[10,16,17]$ and $R_{D^{*}}[8-10,16,17]$ exceed the SM predictions by about $1.4 \sigma$ and $2.5 \sigma$ respectively $[18,19]$, and their combination leads to a deviation of $3.1 \sigma$ from the SM prediction [16, 17, 20, 21]. On the other hand, and independently of the charged current modes, the measurement of $R_{K}$ for the dilepton invariant mass squared bin $[1.1,6] \mathrm{GeV}^{2}[11]$ displays a $2.5 \sigma$ deviation below the SM prediction [22, 23]. Likewise, the measurement of $R_{K^{*}}$ [12] translates into $2.3 \sigma$ and $2.6 \sigma$ deviations, also below the expected SM values for the dilepton invariant mass squared bins $[0.045,1.1] \mathrm{GeV}^{2}$ and $[1.1,6] \mathrm{GeV}^{2}$, respectively $[22,23]$.

Further neutral current anomalies have emerged, for instance in the observable $\Phi \equiv$ $d \operatorname{BR}\left(B_{s} \rightarrow \phi \mu \mu\right) / d m_{\mu \mu}^{2}$, in a similar kinematic regime $\left(m_{\mu \mu}^{2} \in[1,6] \mathrm{GeV}^{2}\right)[14,24,25]$, also with a deviation of about $3 \sigma$. Deviations from the SM expectations have also been found in the angular observable $P_{5}^{\prime}$ of the $B \rightarrow K^{*} \ell^{+} \ell^{-}$decay.

Although these anomalies by no means invalidate the SM at this stage, their persistence and relatively coherent pattern inevitably raise the question of which (minimal) new ingredients beyond the SM (BSM) would be required to explain them. A first modelindependent approach [19, 20, 26-39] is to introduce higher dimensional effective operators, coupling two quarks with two leptons. Despite the large number of possibilities, it is nevertheless remarkable that only a reduced number of such non-standard couplings significantly eases the tensions with the SM predictions.

It is thus desirable to consider which BSM constructions could be at the origin of these effective operators. Among the most minimal scenarios studied, one has flavour-sensitive $Z^{\prime}$ exchanges [40-53], leptoquark exchanges [54-79], $R$-parity violating supersymmetric models [80-85], and various other constructions [86-95].

In this work, we focus on the exchange of a vector leptoquark $V_{1}$ transforming as $(\mathbf{3}, \mathbf{1}, 2 / 3)$ under the SM gauge group, which has been shown to be particularly attractive for its ability to provide a single particle solution simultaneously to both charged and neutral current anomalies [96-108]. Complying with the experimental measurements suggests that $V_{1}$ should have non-universal couplings to quarks and leptons. We assume $V_{1}$ to be an elementary spin-1 gauge boson; since it carries charges (as a leptoquark must), the underlying gauge symmetry is necessarily non-abelian, with universal (gauge) couplings as long 
as it remains unbroken. ${ }^{1}$ As an example, such a field $V_{1}$ is naturally contained within the theoretically well-motivated Pati-Salam model (PS) as an SU(4) gauge boson. However, the current bounds on the charged lepton flavour violating (cLFV) decays $K_{L} \rightarrow \mu e$ and $K \rightarrow \pi \mu e$ lead to dramatic (lower) bounds on the mass of such a vector leptoquark $\left(m_{V}\right)$, typically above the $100 \mathrm{TeV}$ scale for $\mathcal{O}(1)$ couplings [110-115]. In turn, this renders the new state excessively heavy to account for the $B$-meson decay anomalies.

The cLFV bound on $m_{V}$ turns out to effectively preclude a viable solution to both charged and neutral current anomalies: in the unbroken phase, $V_{1}$ has a single universal coupling to matter; $\mathrm{SU}(2)$-breaking introduces a possible misalignment of the quark and lepton mass eigenbases, thus resulting in LFU-violating $V_{1}$ couplings, proportional to a $3 \times 3$ unitary matrix. In order to explain the $R_{D^{(*)}}$ anomalies, the $b \tau$ and $s \tau$ couplings are required to be large, ${ }^{2}$ which in turn leads to large couplings between the first two generations of quarks and leptons (as a consequence of the unitarity of the mixing matrix), leading to excessive contributions to cLFV. The question that naturally emerges is whether one can find a minimal embedding of $V_{1}$ that successfully allows to overcome the cLFV constraints and address both $R_{K^{(*)}}$ and $R_{D^{(*)}}$ anomalies. In other words, can one go beyond the tight constraints arising from a $(3 \times 3)$ unitary mixing of quarks and leptons? This necessarily requires the addition of new fields, beyond $V_{1}$, and along these lines, one possibility is to add other vector leptoquark fields (thus implying a larger gauge group), whose mixing would allow to overcome the above mentioned constraints, as explored in [99].

In the present study, we avoid this further enlarging of the gauge group, adhering to the single vector leptoquark hypothesis, and pursue a distinct avenue. In particular, and motivated by the phenomenological impact of having nonunitary left-handed leptonic mixings in the presence of (heavy) sterile neutral leptons [117-120], we consider the possibility of nonunitary $V_{1}$ couplings, as arising from the presence of $n$ additional vector-like heavy leptons $L$ (also present in the construction of [99]). In the broken phase, the $V_{1}$ couplings are then given by a $(3+n) \times(3+n)$ mixing matrix, so that the couplings to SM fermions now correspond to a $3 \times 3$ sub-block, which is no longer unitary. We argue that this departure from unitary mixings might indeed hold the key to simultaneously address $R_{K^{(*)}}$ and $R_{D^{(*)}}$ data, while satisfying existing cLFV constraints.

The addition of vector-like heavy charged leptons ${ }^{3}$ can be seen as an intermediary step towards a full ultraviolet-complete model, providing a better framework for the pecu-

\footnotetext{
${ }^{1}$ In this work we are interested in the minimal (gauge extension) scenario where the vector leptoquark is an elementary gauge boson corresponding to a gauge group under which the SM fermion generations are universally charged and no additional protection or symmetry is introduced to induce non-universality. For models in which the vector leptoquark appears as a composite field, see for instance [109]; for other models where the gauge group is non-minimal and/or the gauge charges of the SM fermion generations are non-universal, see e.g. [70].

${ }^{2}$ To satisfy the constraints from the $\tau$ decays, the $c \nu$ coupling induced by $b \tau$ via CKM mixing is in general not sufficient to comfortably explain $R_{D^{(*)}}$ [116]; on the other hand the maximum $c \nu$ coupling induced by $d_{i} \mu$ and $d_{i} e$ (for neutrino flavour in $c \nu$ different from $\nu_{\tau}$ ) are fixed by $R_{K}^{(*)}$ data (for $i=2,3$ ) and kaon decays (for $i=1$ ), while the $c \nu$ coupling induced by $d \tau$ is highly CKM suppressed. In view of this and working in a unitary parametrisation of the leptoquark couplings, the only viable possibility therefore is to maximise the $b \tau$ and $s \tau$ entries.

${ }^{3}$ Heavy vector-like quarks will not be considered, as they are not required for a minimal working model.
} 
liar structure of leptoquark couplings required by the anomalies. In this framework, the nonunitary mixings will also lead to the modification of SM-like charged and neutral lepton currents, establishing an inevitable link to electroweak precision (EWP) observables, such as lepton flavour violating and/or LFUV $Z$-decays. The latter observables will prove to be extremely constraining, ultimately leading to the exclusion of isosinglet vector-like heavy leptons as a source of non-universality in $B$-meson decays.

These constraints are much milder for isodoublet heavy leptons: after arguing that for a single additional heavy charged lepton, cLFV constraints exclude an explanation of even $R_{D^{(*)}}$ alone, we show that the addition of $n=3$ vector-like isodoublet leptons allows a simultaneous explanation of both $R_{K^{(*)}}$ and $R_{D^{(*)}}$ anomalies, while respecting all available constraints.

This work is organised as follows: in section 2 we describe the underlying framework; section 3 is devoted to a comprehensive analysis of the phenomenological implications of the nonunitary framework, regarding the $B$-meson anomalies, and several flavour and EWP observables. A summary and concluding remarks can be found in section 4 .

\section{Towards a nonunitarity interpretation of vector leptoquark couplings}

As mentioned in the Introduction, we consider here a SM extension by a single vector leptoquark $V_{1}$, which transforms under the $\mathrm{SM}$ gauge group $\mathrm{SU}(3)_{c} \times \mathrm{SU}(2)_{L} \times \mathrm{U}(1)_{Y}$ as $(\mathbf{3}, \mathbf{1}, 2 / 3)$. Without loss of generality, we assume that $V_{1}$ is a gauge boson of an unspecified gauge extension of $\mathrm{SU}(3)_{c}$ with a universal (i.e. flavour blind) gauge coupling; without relying on a specific gauge embedding and/or Higgs sector, our only working assumption is that all fermions acquire a mass after electroweak symmetry breaking (EWSB), and that the physical eigenstates are obtained from the diagonalisation of the corresponding (generic) mass matrices. In the weak basis, the interaction of $V_{1}$ with the SM matter fields can be written as

$$
\mathcal{L} \supset \sum_{i=1}^{3} V_{1}^{\mu}\left[\frac{\kappa_{L}}{\sqrt{2}}\left(\bar{d}_{L}^{0, i} \gamma_{\mu} \ell_{L}^{0, i}+\bar{u}_{L}^{0, i} \gamma^{\mu} \nu_{L}^{0, i}\right)+\frac{\kappa_{R}}{\sqrt{2}} \bar{d}_{R}^{0, i} \gamma_{\mu} \ell_{R}^{0, i}+\frac{\bar{\kappa}_{R}}{\sqrt{2}} \bar{u}_{R}^{0, i} \gamma_{\mu} \nu_{R}^{0, i}\right]+\text { H.c. }
$$

in which the " 0 " superscript denotes interaction states, and $i=1-3$ are family indices. The couplings $\kappa_{L, R}$ are flavour diagonal, and universal. Since left-handed couplings are the minimal essential ingredient frequently called upon to simultaneously explain the neutral and charged current anomalies [97], for simplicity we will henceforth only consider the latter (i.e., taking $\kappa_{L} \neq 0$ and $\kappa_{R}=\bar{\kappa}_{R}=0$ ). Furthermore, notice that this can be easily realised in chiral PS models [97, 104, 105], and is moreover phenomenologically well-motivated. ${ }^{4}$

In terms of physical fields, the Lagrangian can be written as

$$
\mathcal{L} \supset \sum_{i, j, k=1}^{3} V_{1}^{\mu}\left(\bar{d}_{L}^{i} \gamma_{\mu} K_{1}^{i k} \ell_{L}^{k}+\bar{u}_{L}^{j} V_{j i}^{\dagger} \gamma_{\mu} K_{1}^{i k} U_{k j}^{\mathrm{P}} \nu_{L}^{j}\right)+\text { H.c. }
$$

\footnotetext{
${ }^{4}$ In the context of PS unification it has been noted in the literature that if the vector leptoquark couples to both left- and right-handed fermion fields with similar gauge strength, then in the absence of some helicity suppression, bounds from various searches for lepton flavour violating mesonic decay modes put a lower limit on the vector leptoquark mass around $100 \mathrm{TeV}$ [111-115].
} 
where $V$ is the Cabibbo-Kobayashi-Maskawa (CKM) mixing matrix and $U^{\mathrm{P}} \equiv U_{L}^{\ell \dagger} U_{L}^{\nu}$ the Pontecorvo-Maki-Nakagawa-Sakata (PMNS) leptonic mixing matrix; we have also introduced $K_{1} \equiv \frac{\kappa_{L}}{\sqrt{2}} U_{L}^{\ell}$ to denote the "effective" leptoquark couplings in the physical fermion basis. Being proportional to an arbitrary unitary matrix (which we hereby denote $V_{0}$ ), $K_{1}$ can be further cast as

$$
K_{1}=\frac{\kappa_{L}}{\sqrt{2}} V_{0}=\frac{\kappa_{L}}{\sqrt{2}}\left(\begin{array}{ccc}
c_{12} c_{13} & s_{12} c_{13} & s_{13} \\
-s_{12} c_{23}-c_{12} s_{23} s_{13} & c_{12} c_{23}-s_{12} s_{23} s_{13} & s_{23} c_{13} \\
s_{12} s_{23}-c_{12} c_{23} s_{13} & -c_{12} s_{23}-s_{12} c_{23} s_{13} & c_{23} c_{13}
\end{array}\right)
$$

in which we used the standard parametrisation of a real $3 \times 3$ unitary matrix in terms of three angles $\theta_{12,23,13}$ (with $c_{i j}$ and $s_{i j}$ respectively denoting $\cos \theta_{i j}$ and $\sin \theta_{i j}$ ), and we restrict ourselves to real parameter space in all our analysis. (We emphasise here that $V_{0}$ is not the PMNS matrix, and that the above angles are not those associated with neutrino oscillation data.)

\subsection{Accounting for $R_{K^{(*)}}$ and $R_{D^{(*)}}$ in a minimal $V_{1}$ leptoquark framework}

The presence of a vector leptoquark, whose interactions with quarks and leptons are defined in eqs. (2.1), (2.2), can induce new operators, contributing to $b$-decays (both neutral and charged currents). In the SM, $b \rightarrow s \ell \ell$ and $b \rightarrow c \ell \nu$ decays respectively occur at one-loop and at tree-level; on the other hand, the new $V_{1}$-mediated contributions to both decays arise at the tree-level. Thus, the new contributions required to explain $R_{K^{(*)}}$ data are comparatively smaller than those needed to account for the discrepancy in $R_{D^{(*)}}$ data: in particular, $R_{D^{(*)}}$ requires the mass scale of $V_{1}$ to be quite low $\sim \mathcal{O}(1 \mathrm{TeV})$, while it is possible to explain $R_{K^{(*)}}$ for leptoquark masses $m_{V} \sim \mathcal{O}(10 \mathrm{TeV})$ (taking into account all the constraints from rare transitions and decays). The low mass scale required to explain the $R_{D^{(*)}}$ anomaly effectively precludes a simultaneous (combined) explanation for both anomalies, due to the excessive associated contributions to cLFV kaon decays, in particular to $K_{L} \rightarrow e^{ \pm} \mu^{\mp}$ (which occurs at the tree level). Consequently, both modes $\left(K_{L} \rightarrow e^{+} \mu^{-}\right.$ and $K_{L} \rightarrow e^{-} \mu^{+}$) have to be suppressed separately. In terms of the parametrisation of eq. (2.3), saturating $R_{D^{(*)}}$ requires maximising the 23 and 33 entries of $V_{0}$ (thus leading to $\theta_{13} \sim 0$ and $\left.\theta_{23} \sim \frac{\pi}{4}\right)$. This implies that the branching fractions of the tree-level kaon decay modes are proportional to $\sin ^{2} \theta_{12}$ and $\cos ^{2} \theta_{12}$, respectively. A sufficient and simultaneous suppression of contributions to these modes is then clearly not possible.

Likewise, excessively large contributions to $\mu-e$ conversion (also occurring at tree-level) further exclude a low scale realisation, with $m_{V} \sim \mathcal{O}(1 \mathrm{TeV})$. The above arguments are illustrated by figure 1 , in which we display the predictions for neutrinoless $\mu-e$ conversion and $K_{L} \rightarrow e^{ \pm} \mu^{\mp}$ associated with having contributions to $R_{D^{(*)}}$ within $3 \sigma$ of the current best fit (for $m_{V} \sim \mathcal{O}\left(1 \mathrm{TeV}\right.$ ) and three different values of $\kappa_{L} / \sqrt{2}$ ).

\subsection{Vector-like fermions and "effective" nonunitary mixings in the light sector}

The above discussion suggests that the minimal flavour structure encoded in the (unitary) parametrisation of the leptoquark-quark-lepton currents (eq. (2.2)) is insufficient to account 


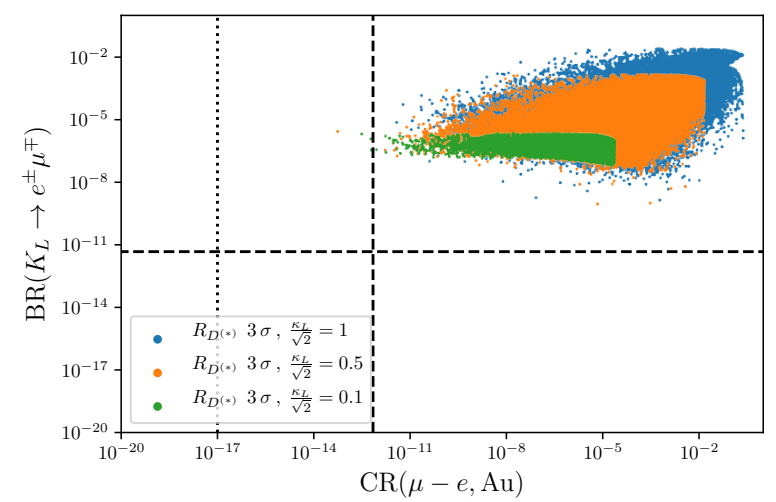

Figure 1. Associated predictions for $\mathrm{CR}(\mu-e, \mathrm{Au})$ and $\mathrm{BR}\left(K_{L} \rightarrow e^{ \pm} \mu^{\mp}\right)$ for sample points satisfying $R_{D^{(*)}}$ at the $3 \sigma$ level for the (unitary) parametrisation of eq. (2.3). The dashed lines represent the current experimental upper bounds (see tables 1 and 3 in section 3.2), and the dotted line a benchmark future sensitivity to $\mathrm{CR}(\mu-e, \mathrm{Al})$. All mixing angles have been varied randomly between $-\pi$ and $\pi$ and the leptoquark mass is set to $m_{V} \sim 1.5 \mathrm{TeV}$. The blue, orange and green points respectively correspond to three benchmark choices, $\frac{\kappa_{L}}{\sqrt{2}}=1,0.5,0.1$.

for both anomalies. A stronger enhancement of LFUV in the leptoquark couplings can be achieved if one hypothesises that the "effective" leptoquark mixings - i.e. the $3 \times 3$ matrix $V_{0}$ is nonunitary. As we proceed to discuss, in order to explain $R_{K^{(*)}}$ and $R_{D^{(*)}}$ data simultaneously, and for universal gauge couplings, a highly nonunitary flavour misalignment between quarks and leptons is in fact required.

Such a nonunitary flavour misalignment can be understood in the presence of heavy vector-like fermions, $\mathrm{SU}(2)_{L}$ singlets or doublets, which have non-negligible mixings with the SM fermions. This can be encoded by generalising the charged lepton mixing matrix to a $3 \times(3+n)$ semi-unitary matrix, so that SM interaction fields and physical states are related as $\ell_{L}^{0}=U_{L}^{\ell} \ell_{L}$ (for $n$ additional heavy states).

The Lagrangian of eq. (2.2) can thus be recast as

$$
\mathcal{L} \supset \sum_{i, j=1}^{3} \sum_{k=1}^{3+n} V_{1}^{\mu}\left(\bar{d}_{L}^{i} \gamma_{\mu} K_{L}^{i k} \ell_{L}^{k}+\bar{u}_{L}^{j} V_{j i}^{\dagger} \gamma_{\mu} K_{L}^{i k} U_{k j}^{\mathrm{P}} \nu_{L}^{j}\right)+\text { H.c. }
$$

Notice that in the above equation, the effective leptoquark coupling $K_{L}$ generalises $K_{1}$ of eq. (2.3), and now corresponds to a rectangular $3 \times(3+n)$ matrix, which can be written in terms of $U_{L}^{\ell}$ as $K_{L} \equiv \frac{\kappa_{L}}{\sqrt{2}} U_{L}^{\ell}$.

Finally, $K_{L}$ can be further decomposed as $K_{L}=\left(K_{1}, K_{2}\right)$, so that $K_{1}$ can be now identified with the nonunitary mixings in the light sectors (contrary to the simple limit of eq. (2.3)). $K_{2}$ is a $3 \times n$ matrix which corresponds to the $n$ heavy degrees of freedom describing the coupling parameters of the heavy (vector-like) states. Inspired by the approach frequently adopted in the context of neutrino physics, the deviation from unitarity 
in the $K_{1}$ block can now be parametrised as [117-120]

$$
K_{1}=\frac{\kappa_{L}}{\sqrt{2}} A V_{0}=\frac{\kappa_{L}}{\sqrt{2}}\left(\begin{array}{ccc}
\alpha_{11} & 0 & 0 \\
\alpha_{21} & \alpha_{22} & 0 \\
\alpha_{31} & \alpha_{32} & \alpha_{33}
\end{array}\right) V_{0},
$$

with $V_{0}$ given in eq. (2.3). The left-triangle matrix $A$, characterises the deviation from unitarity and encodes the effects of the mixings with the heavy states.

As already mentioned in the Introduction, we assume that the vector leptoquark $V_{1}$ appears as a gauge boson in an unspecified $\mathrm{SU}(3)_{c}$ extension. Since neither the gauge embedding nor the Higgs sector is explicitly specified, our only assumption is that after EWSB all fermions (SM and vector-like) are massive, and that the physical eigenstates are obtained from the diagonalisation of an (effective) generic $(3+n) \times(3+n)$ lepton mass matrix. For simplicity (see section 3.3), we take $n=3$ generations of heavy leptons in what follows; the $6 \times 6$ charged lepton mass matrix $\mathcal{M}_{\ell}$ can be diagonalised by a bi-unitary transformation

$$
\mathcal{M}_{\ell}^{\text {diag }}=U_{L}^{\ell \dagger} \mathcal{M}_{\ell} U_{R}^{\ell}
$$

Being a unitary $6 \times 6$ matrix, $U_{L}^{\ell}$ can be parametrised by 15 real angles and 10 phases, and cast as a the product of 15 unitary rotations, $\mathcal{R}_{i j}$. By choosing a convenient ordering for the products of the complex rotation matrices, one can establish a parametrisation that allows isolating the information relative to the heavy leptons in a simple and compact form. Schematically, this can be described by the following $(2 \times 2$ block matrix $)$ decomposition [117], to which we adhere for the remainder of our discussion,

$$
U_{L}^{\ell}=\left(\begin{array}{ll}
A & R \\
B & S
\end{array}\right)\left(\begin{array}{ll}
V_{0} & \mathbf{0} \\
\mathbf{0} & \mathbf{1}
\end{array}\right)
$$

further defining

$$
\begin{aligned}
& \left(\begin{array}{cc}
A & R \\
B & S
\end{array}\right)=\mathcal{R}_{56} \mathcal{R}_{46} \mathcal{R}_{36} \mathcal{R}_{26} \mathcal{R}_{16} \mathcal{R}_{45} \mathcal{R}_{35} \mathcal{R}_{25} \mathcal{R}_{15} \mathcal{R}_{34} \mathcal{R}_{24} \mathcal{R}_{14} \\
& \left(\begin{array}{cc}
V_{0} & \mathbf{0} \\
\mathbf{0} & \mathbf{1}
\end{array}\right)=\mathcal{R}_{23} \mathcal{R}_{13} \mathcal{R}_{12} .
\end{aligned}
$$

Under the above decomposition, one can still identify the SM-like mixings, given by $V_{0}$ (cf. eq. (2.3)); the leptoquark couplings ${ }^{5}$ are now parametrised by the $3 \times 6$ (rectangular) matrix,

$$
K_{L}=\left(K_{1}, K_{2}\right)=\frac{\kappa_{L}}{\sqrt{2}}\left(A V_{0}, R\right) .
$$

The diagonal elements of the triangular matrix $A, \alpha_{i i}$, can be expressed as

$$
\alpha_{i i}=c_{i 6} c_{i 5} c_{i 4},
$$

\footnotetext{
${ }^{5}$ Note that this is an identification of the mixing elements with the effective leptoquark couplings by choosing the basis in which the down-type quarks are diagonal.
} 
in terms of the cosines of the mixing angles, $c_{i j}=\cos \theta_{i j}$. (The SM-like limit can be recovered for $A \rightarrow \mathbf{1}$.) The off-diagonal elements can be cast as [117]

$$
\begin{aligned}
\alpha_{21}= & -c_{14} c_{15} \hat{s}_{16} \hat{s}_{26}^{*}-c_{14} \hat{s}_{15} \hat{s}_{25}^{*} c_{26}-\hat{s}_{14} \hat{s}_{24}^{*} c_{25} c_{26}, \\
\alpha_{32}= & -c_{24} c_{25} \hat{s}_{26} \hat{s}_{36}^{*}-c_{24} \hat{s}_{25} \hat{s}_{35}^{*} c_{36}-\hat{s}_{24} \hat{s}_{34}^{*} c_{35} c_{36}, \\
\alpha_{31}= & -c_{14} c_{15} \hat{s}_{16} c_{26} \hat{s}_{36}^{*}+c_{14} \hat{s}_{15} \hat{s}_{25}^{*} \hat{s}_{26} \hat{s}_{36}^{*}-c_{14} \hat{s}_{15} c_{25} \hat{s}_{35}^{*} c_{36} \\
& +\hat{s}_{14} \hat{s}_{24}^{*} c_{25} \hat{s}_{26} \hat{s}_{36}^{*}+\hat{s}_{14} \hat{s}_{24}^{*} \hat{s}_{25} \hat{s}_{35}^{*} c_{36}-\hat{s}_{14} c_{24} \hat{s}_{34}^{*} c_{35} c_{36},
\end{aligned}
$$

where $\hat{s}_{i j} \equiv e^{i \delta_{i j}} \sin \theta_{i j}$, with $\theta_{i j}$ and $\delta_{i j}$ respectively being the angles and CP phases associated with the $\mathcal{R}_{i j}$ rotation. Finally, it is worth emphasising that not only the full $6 \times 6$ matrix $U_{L}^{\ell}$ is unitary, but its upper $3 \times 6$ block $\left(A V_{0}, R\right)$ is also semi-unitary on its own, with $\frac{2}{\kappa_{L}^{2}} K_{L} K_{L}^{\dagger}=1$.

This formalism, which can be easily generalised to $n$ extra generations, offers the possibility of successfully separating the information relative to the heavy leptons in a simple and compact form. Although the couplings (in particular the $\alpha_{i j}$ entries) can be in general complex, in what follows we consider a minimal scenario where all couplings are taken to be real.

\section{Explaining LFUV data with nonunitary couplings: phenomenological viability}

We recall that, as mentioned in the Introduction, we work under the minimal assumptions that the singlet vector leptoquark $V_{1}$ (colour triplet) should correspond to a gauge extension of $\mathrm{SU}(3)_{c}$ unifying quarks and leptons with a universal (i.e., flavour independent) gauge coupling. We first describe the effects of the vector leptoquark on the neutral and charged current $b$ decays, and then summarise the most stringent constraints arising from numerous flavour violating and flavour conserving observables (meson oscillations and decays, as well as charged lepton flavour violation processes). We then present our main numerical results.

\subsection{New contributions to $\boldsymbol{R}_{K^{(*)}}$ and $\boldsymbol{R}_{D^{(*)}}$}

In what follows, we proceed to explore whether the relaxation of the unitarity requirement on the $\mathrm{SU}(2)_{L}$-singlet vector leptoquark $V_{1}$ couplings to $\mathrm{SM}$ matter does allow addressing $R_{K^{(*)}}$ and $R_{D^{(*)}}$ data simultaneously.

Anomalies in neutral current $\boldsymbol{b}$ decays: $\boldsymbol{R}_{\boldsymbol{K}^{(*)}}$. As mentioned in the Introduction, several measurements of the ratio of branching ratios of $B \rightarrow K^{(*)} \ell \ell(\ell=e, \mu)$ exhibit tensions when compared to the SM predictions. The most recent averages (and SM estimations) are associated with the following deviations [11-13], in which the dilepton invariant mass squared bin (in $\mathrm{GeV}^{2}$ ) is identified by the subscript:

$$
\begin{aligned}
R_{K[1.1,6]}^{\mathrm{LHCb}} & =0.846 \pm_{0.054}^{0.060} \pm_{0.014}^{0.016}, & R_{K}^{\mathrm{SM}} & =1.0003 \pm 0.0001, \\
R_{K^{*}[0.045,1.1]}^{\mathrm{LHCb}} & =0.66_{-0.07}^{+0.11} \pm 0.03, & R_{K^{*}[0.045,1.1]}^{\text {Belle }} & =0.52_{-0.26}^{+0.36} \pm 0.05, \\
R_{K^{*}[1.1,6]}^{\mathrm{LHCb}} & =0.69_{-0.07}^{+0.11} \pm 0.05, & R_{K^{*}[0.045,1.1]}^{\mathrm{SM}} & \sim 0.93, \\
R_{K^{*}[1,6]}^{\text {Bele }} & =0.96_{-0.29}^{+0.45} \pm 0.11, & R_{K^{*}[1.1,6]}^{\mathrm{SM}} & \sim 0.99 .
\end{aligned}
$$


Other anomalies in the neutral current mode of $B$ meson decays have also emerged concerning the observable $\Phi \equiv d \operatorname{BR}\left(B_{s} \rightarrow \phi \mu \mu\right) / d m_{\mu \mu}^{2}$ in the analogous bin $\left(m_{\mu \mu}^{2} \in\right.$ $[1,6] \mathrm{GeV}^{2}$ ) [14], with a similar deviation (at a level of approximately $3 \sigma$ ), as well as in the angular observable $P_{5}^{\prime}$ in $B \rightarrow K^{*} \ell^{+} \ell^{-}$processes. While LHCb's results for $P_{5}^{\prime}$ in $B \rightarrow K^{*} \mu^{+} \mu^{-}$decays manifest a slight discrepancy with respect to the SM, the Belle Collaboration [15] reported that, when compared to the muon case, $P_{5}^{\prime}$ results for electrons show a better agreement with theoretical SM expectations. Nevertheless, there has been an ongoing discussion about the possibility that incorrectly estimated hadronic uncertainties might be at the origin of the observed anomalies in the mode $B \rightarrow K^{*} \mu^{+} \mu^{-}$, due to power corrections to the form factors, or charm-loop contributions [123-126].

The effective Hamiltonian describing the neutral current effects at the level of quark transitions $d_{j} \rightarrow d_{i} \ell^{-} \ell^{\prime+}$ is given in appendix A. A model-independent analysis of the current data at the $b$-quark mass scale can be made using the $2 \mathrm{D}$ hypothesis $C_{9, \mathrm{NP}}^{\mu \mu}=$ $-C_{10, \mathrm{NP}}^{\mu \mu}$ and $C_{9, \mathrm{NP}}^{e e}=-C_{10, \mathrm{NP}}^{e e}$, allowing for the possibility of $(V-A)$ new physics (NP) effects in both electron and muon channels. Using the available experimental measurements of $R_{K^{(*)}}$ in different high and low $q^{2}$ bins, including the latest updates from LHCb and Belle collaborations, the available experimental measurements on the angular observables for $b \rightarrow s \mu \mu$ and $b \rightarrow$ see, and the latest average for $\operatorname{BR}\left(B_{s} \rightarrow \mu \mu\right)$ - which already includes the latest measurement from the ATLAS collaboration — we find the global fit ranges

$$
\begin{aligned}
-0.50(-0.58) & \geq C_{9, s b}^{\mu \mu}=-C_{10, s b}^{\mu \mu} \geq(-0.83)-0.91, \\
0.00(-0.12) & \geq C_{9, s b}^{e e}=-C_{10, s b}^{e e} \geq(-0.45)-0.55,
\end{aligned}
$$

at the $2 \sigma(1 \sigma)$ level. In figure 2 we show the $1 \sigma$ likelihood contours from the latest experimental measurements of $R_{K}^{(*)}, b \rightarrow s \mu \mu$ and $b \rightarrow$ see observables, as well as their combined global fit $(1 \sigma$ and $2 \sigma)$ in the $C_{9, s b}^{\mu \mu}$ vs. $C_{9, s b}^{e e}$ plane, assuming the $2 \mathrm{D}$ hypothesis $C_{9, s b}^{\mu \mu}=-C_{10, s b}^{\mu \mu}$ and $C_{9, s b}^{e e}=-C_{10, s b}^{e e}$.

The vector leptoquark $V_{1}$ contributes at tree level, yielding the following Wilson coefficients at the leptoquark mass scale for the $\mathcal{O}_{9,10}^{i j ; \ell^{\prime}}$ operators [127]

$$
C_{9}^{i j ; \ell \ell^{\prime}}=-C_{10}^{i j ; \ell \ell^{\prime}}=-\frac{\pi}{\sqrt{2} G_{F} \alpha_{\mathrm{em}} V_{3 j} V_{3 i}^{*}} \frac{1}{m_{V}^{2}} K_{L}^{i \ell^{\prime}} K_{L}^{j \ell *}
$$

in which $G_{F}$ denotes the Fermi constant, $\alpha_{\mathrm{em}}$ is the fine-structure constant, $V$ the CKM matrix, $K_{L}$ the "effective" leptoquark couplings (cf. eq. (2.9)) and $m_{V}$ the vector leptoquark mass. The matching of the model parameters with the Wilson coefficients is performed at the scale of leptoquark mass and the Wilson coefficients are subsequently run down to the $b$-quark mass scale [116]. In particular, it is interesting to note that due to RG-running a large $K_{L}^{i \tau}$ coupling can potentially induce a non-negligible lepton-universal contribution to $b \rightarrow$ sll transitions via a log-enhanced anapole photon penguin contribution, as noted in ref. [128]. The dominant log-enhanced contribution is given by

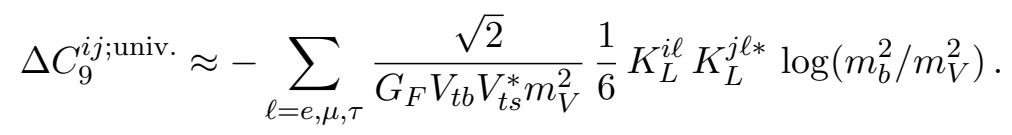




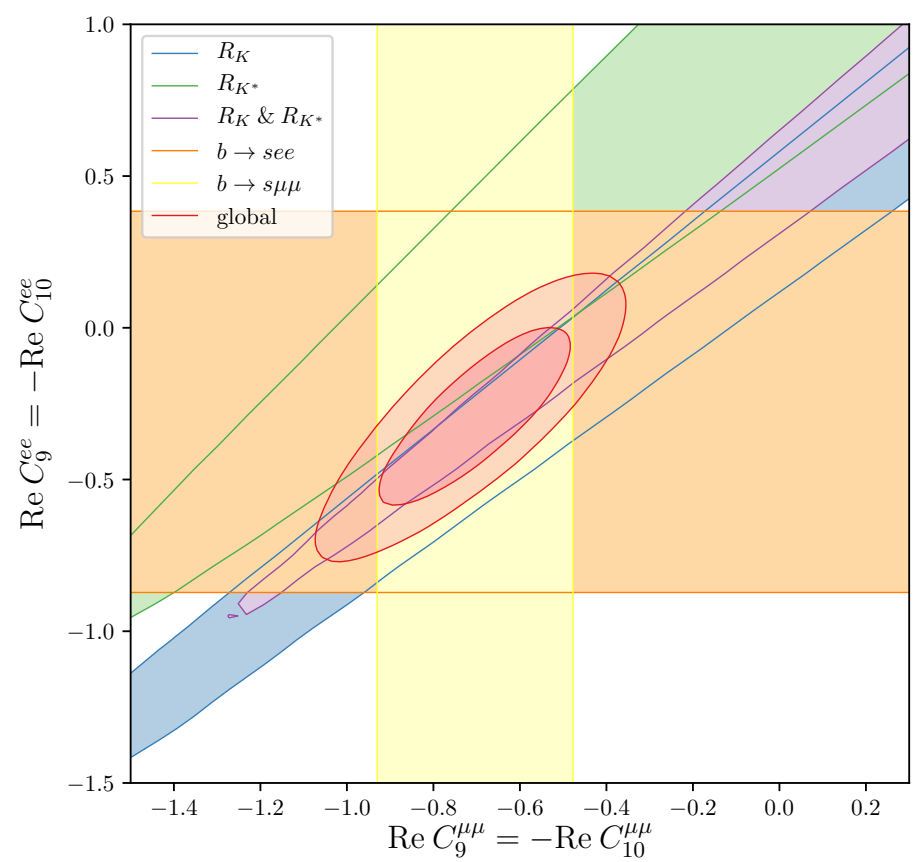

Figure 2. Likelihood $1 \sigma$ contours from the latest measurements of $R_{K}^{(*)}, b \rightarrow s \mu \mu$, and $b \rightarrow$ see observables, as well as the combined global fit $(1 \sigma$ and $2 \sigma)$ in the $C_{9, s b}^{\mu \mu}$ vs. $C_{9, s b}^{e e}$ plane, assuming the $2 \mathrm{D}$ hypothesis $C_{9, s b}^{\mu \mu}=-C_{10, s b}^{\mu \mu}$ and $C_{9, s b}^{e e}=-C_{10, s b}^{e e}$. The global fit is obtained at the $b$-quark mass scale using the package flavio [122].

In our analysis, the running from the scale of leptoquark mass to the $b$-quark mass scale, and to any other relevant process (observable) scale, is taken into account using the wilson package [121] in association with the flavio package [122].

Anomalies in charged current $b \rightarrow \boldsymbol{c} \ell \bar{\nu}$ transitions: $\boldsymbol{R}_{D^{(*)}}$. Important deviations from the SM prediction of lepton flavour universality in $B \rightarrow D^{(*)} \ell \nu$ decays have also been reported by several experimental collaborations. The most recent averages for the $R_{D}$ and $R_{D^{(*)}}$ ratios by the HFLAV Collaboration $[16,17]$ are

$$
\begin{aligned}
R_{D}=0.340 \pm 0.027 \pm 0.013, & R_{D}^{\mathrm{SM}}=0.299 \pm 0.003 \\
R_{D^{*}}=0.295 \pm 0.011 \pm 0.008, & R_{D^{*}}^{\mathrm{SM}}=0.258 \pm 0.005
\end{aligned}
$$

We define the effective Hamiltonian for the charged current transitions $d_{k} \rightarrow u_{j} \bar{\nu} \ell^{-}$as

$$
\mathcal{H}_{\mathrm{eff}}^{\ell_{f} \nu_{i}}=\frac{4 G_{F}}{\sqrt{2}} V_{j k} C_{j k}^{f i}\left(\bar{u}_{j} \gamma^{\mu} P_{L} d_{k}\right)\left(\bar{\ell}_{f} \gamma_{\mu} P_{L} \nu_{i}\right),
$$

where, in the SM, $C_{j k, \mathrm{SM}}^{f i}=\delta_{f i}$. The contribution from the vector leptoquark $V_{1}$ is given by

$$
C_{j k, V_{1}}^{f i}=\frac{\sqrt{2}}{4 G_{F} m_{V}^{2}} \frac{1}{V_{j k}}\left(V K_{L} U^{P}\right)_{j i} K_{L}^{k f *} .
$$

One can further construct the double ratios

$$
R_{D} / R_{D}^{\mathrm{SM}}=R_{D^{*}} / R_{D^{*}}^{\mathrm{SM}}=\sum_{i=1}^{3}\left|\delta_{3 i}+C_{c b, V_{1}}^{\tau i}\right|^{2},
$$




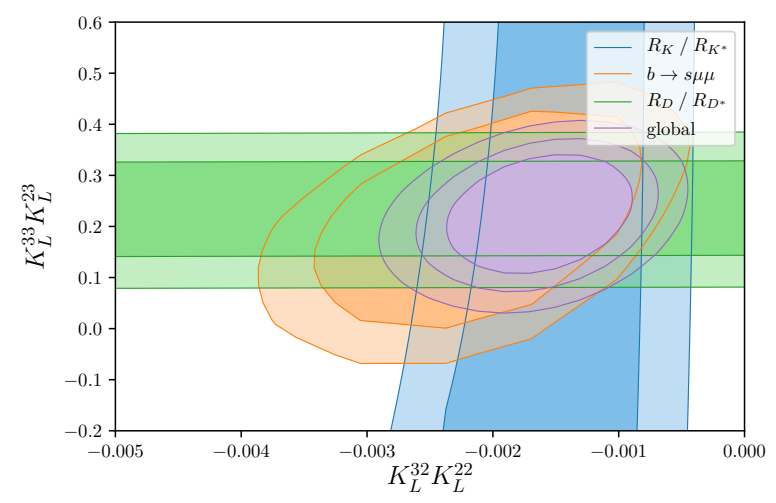

Figure 3. Likelihood $1 \sigma$ and $2 \sigma$ contours from $R_{K^{(*)}}, R_{D^{(*)}}, b \rightarrow s \mu \mu$ observables and the combined global fit $(1 \sigma, 2 \sigma$ and $3 \sigma)$ in the plane $K_{L}^{33} K_{L}^{23}-K_{L}^{32} K_{L}^{22}$, defined at the leptoquark mass scale cf. eq. (2.2). The first generation lepton and quark couplings are set to zero.

(equal to unity when NP decouples, i.e., $\kappa_{L} \rightarrow 0$ and $m_{V} \rightarrow \infty$ ). After combining current experimental world averages with the SM predictions, the current anomalous data can be summarised as $R_{D} / R_{D}^{\mathrm{SM}}=1.14 \pm 0.10, R_{D^{*}} / R_{D^{*}}^{\mathrm{SM}}=1.14 \pm 0.06$, in which the statistical and systematical errors have been added in quadrature. In figure 3 , we display the $1 \sigma$ and $2 \sigma$ likelihood contours from $R_{K^{(*)}}, R_{D^{(*)}}, b \rightarrow s \mu \mu$ observables and the combined global fit $(1 \sigma, 2 \sigma$ and $3 \sigma)$ in the plane $K_{L}^{33} K_{L}^{23}-K_{L}^{32} K_{L}^{22}$, where the couplings (see eq. (2.2)) are varied independently and the others are set to zero.

As can be seen in figure 3 , both $b \rightarrow c \ell \nu$ and $b \rightarrow$ sll anomalies can be indeed accommodated simultaneously in a minimal $V_{1}$ model. However, we will subsequently show how this is realised in the nonunitary framework taking all couplings into account, as suggested by the $b \rightarrow$ sll data.

\subsection{Constraints from (rare) flavour processes, EW precision observables and direct searches}

The extended framework called upon to address the $B$ meson decay anomalies — not only the additional vector leptoquark, but also the presence of extra vector-like fermions, which are the origin of the nonunitarity of the $V_{1}$ effective couplings - opens the door to extensive contributions to numerous observables.

While most of the NP contributions occur via higher order (loop) exchanges, it is important to notice that $V_{1}$ can also mediate very rare (or even SM forbidden) processes already at the tree level. As we proceed to discuss, the latter observables prove to be particularly constraining, and put stringent bounds on the degrees of freedom of these leptoquark realisations.

Leptoquark SM extensions aiming at addressing the anomalies in $R_{K^{(*)}}$ and $R_{D^{(*)}}$ data receive strong constraints from $d_{j} \rightarrow d_{i} \bar{\nu} \nu$ transitions (in particular $s \rightarrow d \nu \nu$ and $b \rightarrow s \nu \nu$ ). However, the vector leptoquark $V_{1}$ does not generate contributions at tree level, and the first non-vanishing contribution appears at one loop. Consequently, we find that even with significant uncertainties, the semileptonic decays into charged dileptons $d_{j} \rightarrow d_{i} \ell^{-} \ell^{\prime+}$ often lead to tighter constraints (both the lepton flavour conserving and the lepton flavour 


\begin{tabular}{|c|c|c|}
\hline Observables & SM prediction & Experimental data \\
\hline $\mathrm{BR}\left(K^{+} \rightarrow \pi^{+} \nu \bar{\nu}\right)$ & $(8.4 \pm 1.0) \times 10^{-11} \quad[129]$ & $\begin{array}{l}17.3_{-10.5}^{+11.5} \times 10^{-11} \quad[130] \\
<11 \times 10^{-10}\end{array}$ \\
\hline $\operatorname{BR}\left(K_{L} \rightarrow \pi^{0} \nu \bar{\nu}\right)$ & $(3.4 \pm 0.6) \times 10^{-11} \quad[129]$ & $\leq 2.6 \times 10^{-8} \quad[132]$ \\
\hline$R_{K^{(*)}}^{\nu \nu}\left(B \rightarrow K^{(*)} \nu \bar{\nu}\right)$ & $R_{K^{(*)}}^{\nu \nu}=1$ & $\begin{array}{ll}R_{K}^{\nu \nu}<3.9 & {[133]} \\
R_{K^{*}}^{\nu \nu}<2.7 & {[133]}\end{array}$ \\
\hline$B_{s}^{0}-\bar{B}_{s}^{0}$ (mixing parameters) & $\begin{array}{l}\Delta_{s}=\left|\Delta_{s}\right| e^{i \phi_{s}}=1 \\
\phi_{s}=0\end{array}$ & $\begin{array}{l}\left|\Delta_{s}\right|=1.01_{-0.10}^{+0.17} \quad[134], \\
\phi_{s}\left[^{\circ}\right]=1.3_{-2.3}^{+2.3} \quad[134]\end{array}$ \\
\hline$K^{0}-\bar{K}^{0}: \Delta m_{K} /\left(10^{-15} \mathrm{GeV}\right)$ & $3.1 \pm 1.2 \quad[135]$ & $3.484 \pm 0.006 \quad[1]$ \\
\hline $\mathrm{BR}\left(B_{s} \rightarrow \mu \mu\right)$ & $(3.23 \pm 0.27) \times 10^{-9} \quad[136]$ & $2.7_{-0.5}^{+0.6} \times 10^{-9} \quad[1]$ \\
\hline $\operatorname{BR}\left(K_{L} \rightarrow \mu^{ \pm} e^{\mp}\right)$ & - & $<4.7 \times 10^{-12} \quad[1]$ \\
\hline $\mathrm{BR}\left(B_{s} \rightarrow \mu^{ \pm} e^{\mp}\right)$ & - & $<1.1 \times 10^{-8} \quad[1]$ \\
\hline $\mathrm{BR}\left(B^{0} \rightarrow \mu^{ \pm} e^{\mp}\right)$ & - & $<2.8 \times 10^{-9} \quad[1]$ \\
\hline $\mathrm{BR}\left(B^{0} \rightarrow \tau^{ \pm} e^{\mp}\right)$ & - & $<2.8 \times 10^{-5}$ \\
\hline $\mathrm{BR}\left(B^{0} \rightarrow \tau^{ \pm} \mu^{\mp}\right)$ & - & $<2.2 \times 10^{-5} \quad[1]$ \\
\hline $\operatorname{BR}\left(B^{0} \rightarrow K^{0} \mu^{ \pm} e^{\mp}\right)$ & - & $<2.7 \times 10^{-8} \quad[1]$ \\
\hline $\operatorname{BR}\left(B^{0} \rightarrow \pi^{0} \mu^{ \pm} e^{\mp}\right)$ & - & $<1.4 \times 10^{-7} \quad[1]$ \\
\hline $\operatorname{BR}\left(B^{ \pm} \rightarrow K^{+} \mu^{ \pm} e^{\mp}\right)$ & - & $<9.1 \times 10^{-8} \quad[1]$ \\
\hline $\operatorname{BR}\left(B^{ \pm} \rightarrow K^{+} \tau^{ \pm} e^{\mp}\right)$ & - & $<3.0 \times 10^{-5} \quad[1]$ \\
\hline $\operatorname{BR}\left(B^{ \pm} \rightarrow K^{+} \tau^{ \pm} \mu^{\mp}\right)$ & - & $<4.8 \times 10^{-5} \quad[1]$ \\
\hline $\mathrm{BR}\left(B^{ \pm} \rightarrow \pi^{+} \mu^{ \pm} e^{\mp}\right)$ & - & $<1.7 \times 10^{-7} \quad[1]$ \\
\hline $\mathrm{BR}\left(B^{ \pm} \rightarrow \pi^{+} \tau^{ \pm} e^{\mp}\right)$ & - & $<7.5 \times 10^{-5} \quad[1]$ \\
\hline $\operatorname{BR}\left(B^{ \pm} \rightarrow \pi^{+} \tau^{ \pm} \mu^{\mp}\right)$ & - & $<7.2 \times 10^{-5} \quad[1]$ \\
\hline
\end{tabular}

Table 1. Relevant observables and current experimental status for leptonic and semi-leptonic meson decays; when appropriate, the associated SM prediction is also included.

violating modes). In the present analysis, we therefore include bounds from $K \rightarrow \pi \ell \ell^{\prime}$ and $B \rightarrow K \ell \ell^{\prime}$, as well as the stringent limits on NP contributions arising from the observed decay mode $B_{s} \rightarrow \mu^{+} \mu^{-}$. Furthermore, we also take into account the lepton flavour violating leptonic decays $B \rightarrow e^{ \pm} \mu^{\mp}, B_{s} \rightarrow e^{ \pm} \mu^{\mp}$ and $K_{L} \rightarrow e^{ \pm} \mu^{\mp}$. Leptoquark contributions to neutral meson oscillations and mixings, such as $K^{0}-\bar{K}^{0}$ and $B_{s}^{0}-\bar{B}_{s}^{0}$, are also evaluated. Table 1 provides a brief summary of the current experimental status for these mesonic observables (current bounds and future sensitivities). A detailed discussion of the formalism used to evaluate the vector leptoquark contributions is provided in appendix A.

The lepton flavour non-universal couplings of vector leptoquarks (in general nonunitary in the present framework) induce new contributions to cLFV observables: radiative decays 
$\ell_{i} \rightarrow \ell_{j} \gamma$ and 3-body decays $\ell_{i} \rightarrow 3 \ell_{j}$ at loop level, and neutrinoless $\mu-e$ conversion in nuclei both at tree and loop level. Further taking into account the impressive associated experimental sensitivity, it is clear that these observables lead to important constraints on the vector leptoquark couplings to SM fermions. It is important to stress that although the radiative decays are generated at higher order, relevant anapole contributions can add to the Wilson coefficients accounting for the tree-level contributions to neutrinoless $\mu-e$ conversion and $\mu \rightarrow 3 e$. The higher order anapole contributions can have a magnitude comparable to the tree level ones (or even account for the dominant contribution). In addition, dipole operators also contribute significantly to radiative decays and to neutrinoless $\mu-e$ conversion. Although we do take tauonic modes into account, we notice here that due to the associated current experimental sensitivity, (semi)leptonic tau decays in general lead to comparatively looser constraints; likewise, semileptonic meson decays into final states including tau leptons are typically less constraining. However, the expected improvements in sensitivity from dedicated experiments might render the tau modes important probes of SM extensions via vector leptoquarks. ${ }^{6}$ As done for the first time in this work, all these contributions must be systematically included to thoroughly constrain the vector leptoquark couplings.

A summary of the current experimental status (current bounds and future sensitivities) is given in table 3; for simplicity, in the numerical analysis we consider a benchmark future sensitivity to $\mu-e$ conversion in Aluminium of $\mathcal{O}\left(10^{-17}\right)$. The relevant details of the computation of the cLFV observables considered in this work can be found in appendix B.

The presence of heavy vector-like fermions (at the source of the nonunitary couplings of the vector leptoquark to the light fermions) can have a non-negligible impact on the couplings of SM fermions to gauge bosons. In turn, this can be manifest in new contributions to several EW precision observables - potentially in conflict with SM expectations and precision data, - which will prove to play a key role in constraining the mixings of the SM charged leptons with the heavy states.

For the $Z$-couplings, which are modified at the tree level, the most stringent constraints are expected to arise from leptonic $Z$ decays; in our analysis, we take into account the LFU ratios and cLFV decay modes of the $Z$ boson. We summarise in table 2 the EWP observables which are of relevance for our study (experimental measurements and SM predictions). A discussion on how the $\bar{f} f Z$ couplings are modified as a consequence of nonunitary effective leptoquark couplings, as well as their impact for several observables, is detailed in appendix $\mathrm{C}$.

Finally, it is clear that the (negative) results of direct searches for the exotic states must be taken into account. At the LHC, pairs of vector leptoquarks can be abundantly produced in various processes (via $t$-channel lepton exchange and direct couplings to one or two gluons). Due to the underlying gauge structure of possible ultraviolet (UV) completions, the production cross section strongly depends on the coupling to gluons which makes the theoretical predictions for the production of vector leptoquarks less robust than those for scalar leptoquarks. On the other hand, if the vector leptoquark corresponds to a

\footnotetext{
${ }^{6}$ For a detailed discussion regarding semileptonic meson decays into final states with tau leptons see, for example, [137].
} 


\begin{tabular}{|c|c|c|}
\hline Observables & Experimental data & SM Prediction \\
\hline$\Gamma_{Z}$ & $2.4952 \pm 0.0023 \mathrm{GeV}$ & $2.4942 \pm 0.0008 \mathrm{GeV}$ \\
$\Gamma\left(Z \rightarrow \ell^{+} \ell^{-}\right)$ & $83.984 \pm 0.086 \mathrm{MeV}$ & $83.959 \pm 0.008 \mathrm{MeV}$ \\
\hline$R_{e}$ & $20.804 \pm 0.050$ & $20.737 \pm 0.010$ \\
$R_{\mu}$ & $20.785 \pm 0.033$ & $20.737 \pm 0.010$ \\
$R_{\tau}$ & $20.764 \pm 0.045$ & $20.782 \pm 0.010$ \\
\hline $\operatorname{BR}\left(Z \rightarrow e^{ \pm} \mu^{\mp}\right)$ & $<7.5 \times 10^{-7}$ & - \\
$\operatorname{BR}\left(Z \rightarrow e^{ \pm} \tau^{\mp}\right)$ & $<9.8 \times 10^{-6}$ & - \\
$\operatorname{BR}\left(Z \rightarrow \mu^{ \pm} \tau^{\mp}\right)$ & $<1.2 \times 10^{-5}$ & - \\
\hline
\end{tabular}

Table 2. Subset of EWP observables affected by the modified $Z$ couplings, with the corresponding experimental measurements and $\mathrm{SM}$ predictions [1]. The ratios $R_{\ell}$ are defined as $R_{\ell}=\Gamma_{\text {had }} / \Gamma\left(Z \rightarrow \ell^{+} \ell^{-}\right)$, and $\Gamma\left(Z \rightarrow \ell^{+} \ell^{-}\right)$denotes an average over $\ell=e, \mu, \tau$.

\begin{tabular}{|c|c|c|}
\hline cLFV process & Current experimental bound & Future sensitivity \\
\hline $\mathrm{BR}(\mu \rightarrow e \gamma)$ & $4.2 \times 10^{-13} \quad($ MEG [138]) & $6 \times 10^{-14} \quad$ (MEG II [139]) \\
\hline $\operatorname{BR}(\tau \rightarrow e \gamma)$ & $3.3 \times 10^{-8} \quad($ BaBar $[140])$ & $10^{-9} \quad$ (Belle II [141]) \\
\hline $\mathrm{BR}(\tau \rightarrow \mu \gamma)$ & $4.4 \times 10^{-8} \quad($ BaBar $[140])$ & $10^{-9} \quad$ (Belle II [141]) \\
\hline $\mathrm{BR}(\mu \rightarrow 3 e)$ & $1.0 \times 10^{-12} \quad($ SINDRUM [142]) & $10^{-15(-16)} \quad($ Mu3e $[143])$ \\
\hline $\mathrm{BR}(\tau \rightarrow 3 e)$ & $2.7 \times 10^{-8} \quad($ Belle [144] $)$ & $10^{-9} \quad$ (Belle II [141]) \\
\hline $\operatorname{BR}(\tau \rightarrow 3 \mu)$ & (Belle [144]) & $10^{-9} \quad($ Belle II [141] $)$ \\
\hline $\mathrm{CR}(\mu-e, \mathrm{~N})$ & $7 \times 10^{-13} \quad(\mathrm{Au}$, SINDRUM [145]) & $10^{-14} \quad(\mathrm{SiC}, \mathrm{DeeMe}[146])$ \\
\hline & & $\begin{array}{l}7 \times 10^{-15}\left(3 \times 10^{-18}\right) \quad(\mathrm{Al}, \mathrm{COMET}[147,148]) \\
8 \times 10^{-17} \quad(\mathrm{Al}, \mathrm{Mu} 2 \mathrm{e}[149])^{7}\end{array}$ \\
\hline
\end{tabular}

Table 3. Current experimental bounds and future sensitivities of various cLFV processes considered in the analysis.

spontaneously broken non-abelian gauge symmetry, gauge invariance completely fixes the couplings between the vector leptoquark and the gluons, implying lower limits on the vector leptoquark mass (albeit still depending on the branching fractions of the leptoquark) from the negative results in the direct searches for pair production at the LHC, see for instance [103]. As a natural consequence of the favoured structure called upon to maximise the effects on $B$-meson decay anomalies, $V_{1}$ is expected to dominantly decay into either $t \bar{\nu}_{\tau}$ or $b \bar{\tau}$. The ATLAS and CMS collaborations have conducted extensive searches, assuming that leptoquarks couple exclusively to third generation quarks and leptons [151-155], which have led to lower limits on the $V_{1}$ mass $\sim 1.5 \mathrm{TeV}$. Further important collider signatures are $p p \rightarrow \tau \bar{\tau}+X$, arising from $t$-channel leptoquark exchange or from single leptoquark production in association with a charged lepton. As argued in $[156,157]$, the projected vector leptoquark reach of HL-LHC with $3 \mathrm{ab}^{-1}$ is close to $1.8 \mathrm{TeV}$. Much higher luminosi-

\footnotetext{
${ }^{7}$ On the long term, and for Titanium targets, Mu2e-II [150] is expected to improve the sensitivity by a factor of ten or more.
} 
ties and/or more sophisticated search strategies are required to probe the preferred mass scale and couplings of states at the origin of a combined explanation of the anomalies (for instance, as suggested by the study of [97]). Other potentially interesting search modes could include $b \mu b \tau$ and $b \mu b \mu$ final states.

In the present analysis, we select (working) benchmark values for the mass and gauge coupling of the vector leptoquark allowing to comply with the current available limits. In particular, for the following numerical analysis we set $\frac{\kappa_{L}}{\sqrt{2}}=1$ as a benchmark choice. Nevertheless, for any other choice consistent with the constraints from direct searches (as discussed above) the qualitative behaviour and the conclusions drawn remain the same. However, for very small values $\kappa_{L}\left(\kappa_{L} \lesssim 0.1\right.$ for $\left.m_{V} \gtrsim 1.5 \mathrm{TeV}\right)$ the number of points in the best fit region for $R_{K^{(*)}}$ and $R_{D^{(*)}}$ anomalies starts to decrease drastically, so that the NP effects become negligible with respect to the SM.

\subsection{Results and discussion}

We now finally address the question of whether a SM extension via vector leptoquarks can simultaneously provide an explanation to both the $R_{K^{(*)}}$ and $R_{D^{(*)}}$ data, working under the hypothesis of universal gauge couplings for the vector leptoquark $V_{1}$. In this framework, the required flavour non-universality arises from nonunitary mixings among the SM fermions - a consequence of the existence of heavy vector-like fermions which have non-negligible mixings with the light fields.

We first begin by considering the most minimal scenario where the nonunitary flavour misalignment is due to the presence of a single generation of heavy vector-like charged leptons (i.e., $n=1$ in eq. (2.4)). Such a minimal field content already leads to a sufficient amount of LFUV to account for both $R_{K^{(*)}}$ and $R_{D^{(*)}}$ anomalies. Although new contributions to rare meson decays and transitions are still in good agreement with current experimental bounds, this scenario is ruled out due to the stringent constraints on $\mathrm{CLFV}^{8}$ modes. Excessive contributions to (tree-level) muon-electron conversion in nuclei play a crucial role in ruling out this realisation, as well as the closely related radiative decays. In order to reconcile the model's prediction with the current bounds on $\mathrm{CR}(\mu-e, \mathrm{Au})$, the photon-penguin contributions must (at least) partially cancel the sizeable tree-level ones; however, such large photon-penguin contributions then translate into unacceptably large $\mu \rightarrow e \gamma$ decay rates, already in conflict with current bounds.

The required flavour non-universality can be recovered for a less dramatic unitarity violation; this can be achieved by extending the particle content by two or more additional heavy charged lepton states, or formally for $n \geq 2$ in eq. (2.4). Although $n=2$ provides more freedom to evade the constraints found in the case $n=1$, no generic solution was found in this case, which might then require an extreme fine tuning to become viable. In the subsequent discussion, we therefore take $n=3$ which is the minimal case and conveniently replicates the number of generations in the SM.

\footnotetext{
${ }^{8}$ Despite being loop-suppressed in the present $V_{1}$ leptoquark SM extension, $B \rightarrow K^{(*)} \nu \bar{\nu}$ decays can in general lead to significant constraints; nonetheless, in the scenarios here discussed, we find that constraints from LFV meson decays, and most importantly cLFV observables, provide tighter constraints.
} 

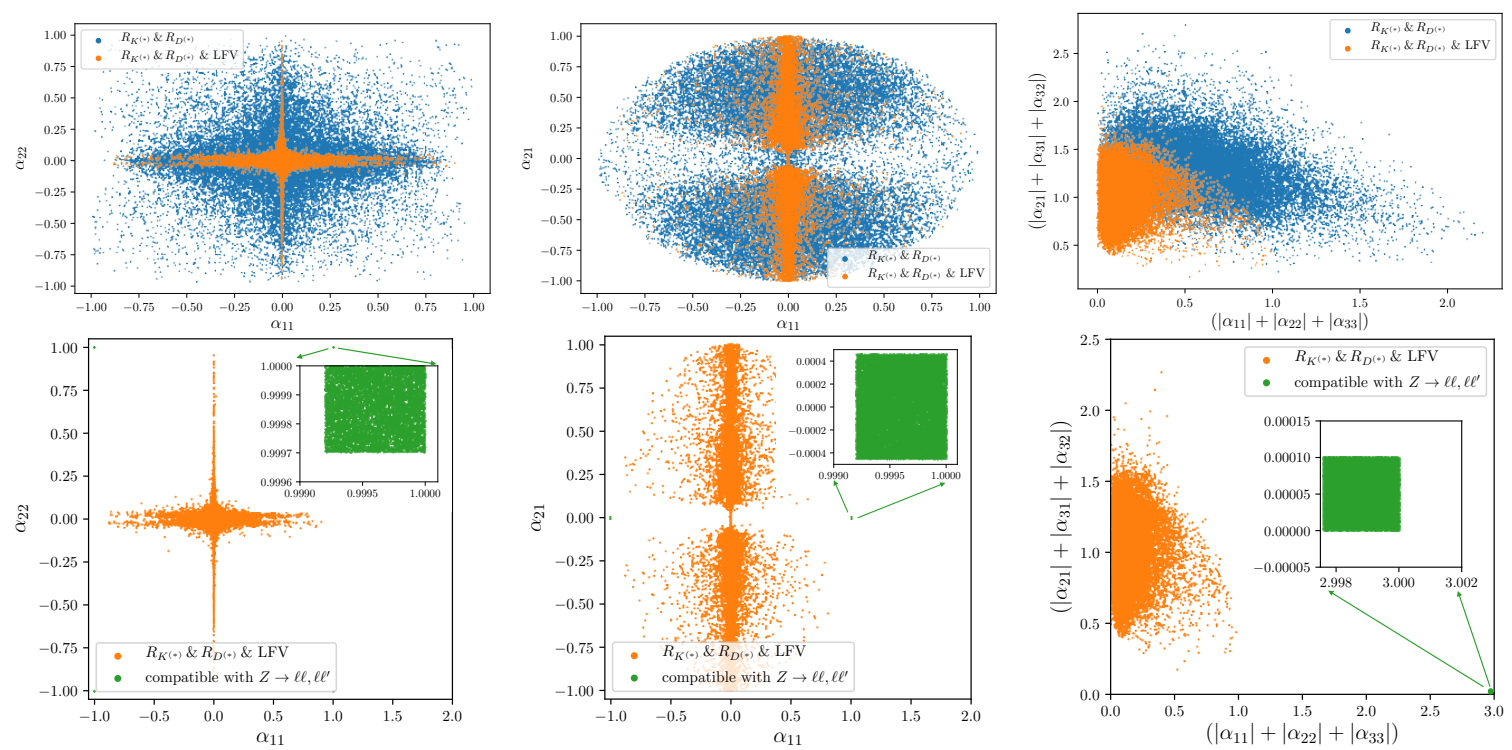

Figure 4. On the upper row, $A$-matrix entries complying with $R_{K^{(*)}}$ and $R_{D^{(*)}}$ data (blue) and those which in addition respect all imposed flavour constraints (yellow). Lower row: for the case of isosinglet heavy leptons, $A$-matrix entries complying with $R_{K^{(*)}}$ and $R_{D^{(*)}}$ data as well as LFV bounds (yellow), and those which now further comply with bounds from $Z$ decays (green). (Notice that the green inset area corresponds to a zoom-out of what would otherwise be a tiny region close to the border of the parameter space.) In all panels we have taken $m_{V}=1.5 \mathrm{TeV}$ and all mixing angles have been varied randomly between $-\pi$ and $\pi$.

For $n=3$, our study suggests that it is in general possible to find regions in the parameter space in which the required non-universal flavour structure to explain both $R_{K^{(*)}}$ and $R_{D^{(*)}}$ arises in a natural way, while still complying with all constraints from flavour violating processes (meson and lepton sectors). This can be seen from the upper row of figure 4 , in which we display the regimes for the entries of the matrix $A$ (cf. eqs. (2.7)-(2.11)) which account for both $R_{K^{(*)}}$ and $R_{D^{(*)}}$ data, as well as regions respecting the constraints arising from the several flavour violating modes considered in our analysis. Concerning the latter, we find it worth mentioning that the most stringent constraints arise from $K_{L} \rightarrow$ $\mu^{ \pm} e^{\mp}, \mu \rightarrow e \gamma$, and $\mu-e$ conversion in nuclei; $B$-meson cLFV decays, or (semi)leptonic $B$ and $K$ decays lead to comparatively milder constraints (or are systematically satisfied).

Finally, one should address the compatibility of the considered SM leptoquark extension with the constraints arising from EW precision tests; as discussed in the previous subsection (and in appendix C), nonunitary mixings can modify the couplings of the $Z$ boson. In particular, for isosinglet heavy leptons, the entries of the matrix $A$ (see eqs. (2.10) and (2.11)) are severely constrained by the $Z$ width and by bounds on its cLFV decays $(Z \rightarrow$ $\ell \ell^{\prime}$ ). This is shown on the lower row of figure 4 , which illustrates the tension between LFUV and $Z$ bounds - a tension which ultimately leads to disfavouring this class of extensions as a phenomenologically viable NP model to explain both $R_{K^{(*)}}$ and $R_{D^{(*)}}$ discrepancies.

If one foregoes a solution to the charged current anomalies (i.e., $R_{D^{(*)}}$ ), it is possible to accommodate $R_{K^{(*)}}$ in full agreement with constraints from flavour bounds, relying on very 

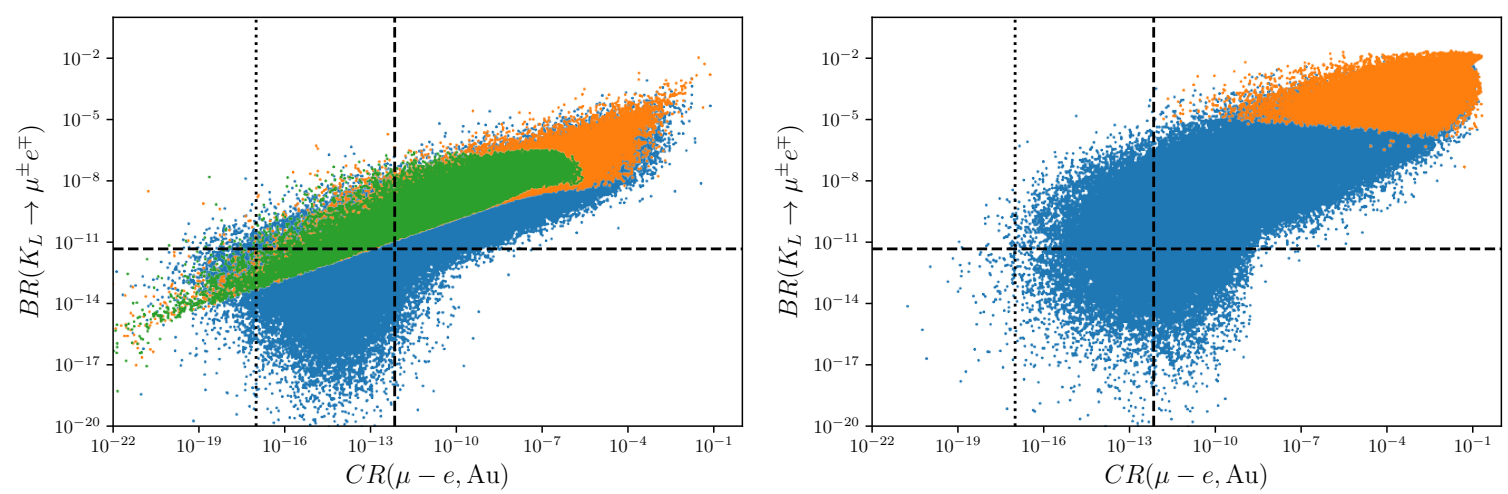

Figure 5. Regions in the plane spanned by $\mathrm{CR}(\mu-e, \mathrm{~N})$ and $\operatorname{BR}\left(K_{L} \rightarrow \mu^{ \pm} e^{\mp}\right)$, accommodating $R_{K^{(*)}}$ (left panel) and $R_{D^{(*)}}$ (right panel), respectively for leptoquark masses in the intervals $m_{V} \in$ [15 TeV, $45 \mathrm{TeV}]$ and $m_{V} \in[1 \mathrm{TeV}, 6 \mathrm{TeV}]$, in the framework of nonunitary leptoquark couplings induced by the presence of 3 generations of isosinglet heavy leptons. Blue points satisfy $R_{K^{(*)}, D^{(*)}}$ at the $3 \sigma$ level, yellow points are consistent with leptonic $Z$ decays, and green points are compatible with all imposed constraints, other than those depicted by the corresponding vertical and horizontal dashed lines (dotted ones denoting future sensitivities). In both panels, all mixing angles have been varied randomly between $-\pi$ and $\pi$.

mild deviations from unitarity, and thus evading constraints from universality violation in $Z$ decays. However, one would be led to regions with considerably heavier leptoquarks, $m_{V} \gtrsim 15 \mathrm{TeV}$. This is depicted on the left panel of figure 5 , in which we display regimes complying with $R_{K^{(*)}}$ at the $3 \sigma$ level) in the plane spanned by two particularly constraining observables, $\mathrm{BR}\left(K_{L} \rightarrow \mu^{ \pm} e^{\mp}\right)$ and $\mathrm{CR}(\mu-e, \mathrm{~N})$, for $15 \mathrm{TeV} \lesssim m_{V} \lesssim 45 \mathrm{TeV}$. As can be verified, a small subset of points (consistent with $R_{K^{(*)}}$ and respecting universality in $Z$ decays) is compatible with current bounds on the cLFV processes. This is in agreement with the analyses of various UV-complete models, such as [104, 105].

For completeness, the right panel of figure 5 shows a similar study for $R_{D^{(*)}}$. In order to accommodate $R_{D^{(*)}}$ data, lower leptoquark masses are required (in this case we have taken $1 \mathrm{TeV} \lesssim m_{V} \lesssim 6 \mathrm{TeV}$ ), and it is no longer possible to evade $K_{L} \rightarrow \mu^{ \pm} e^{\mp}$ and $\mu-e$ conversion bounds while being consistent with leptonic $Z$-decay universality. The data displayed in the panels of figure 4 was obtained for vector leptoquark masses $m_{V} \sim 1.5 \mathrm{TeV}$; analogous conclusions can be inferred for $m_{V} \sim(1-3) \mathrm{TeV}$, albeit for different $\alpha_{i j}$ ranges.

Since for the case of isosinglet leptons an explanation of $R_{D^{(*)}}$ is excluded by bounds on $Z$ decays, we now consider isodoublet heavy charged leptons. The nonunitarity in the couplings of the vector leptoquark to the SM charged leptons can simultaneously explain $R_{K^{(*)}}$ and $R_{D^{(*)}}$ data. Moreover, and by construction, in the case of isodoublet heavy charged lepton states $Z \ell \ell$ couplings remain universal (in the absence of mixings between right-handed SM charged leptons and vector-like doublets, $\Delta g_{R}=0$, see appendix C); nevertheless, flavour observables still play a crucial role, and are (as expected) responsible for severe constraints on the NP degrees of freedom. 

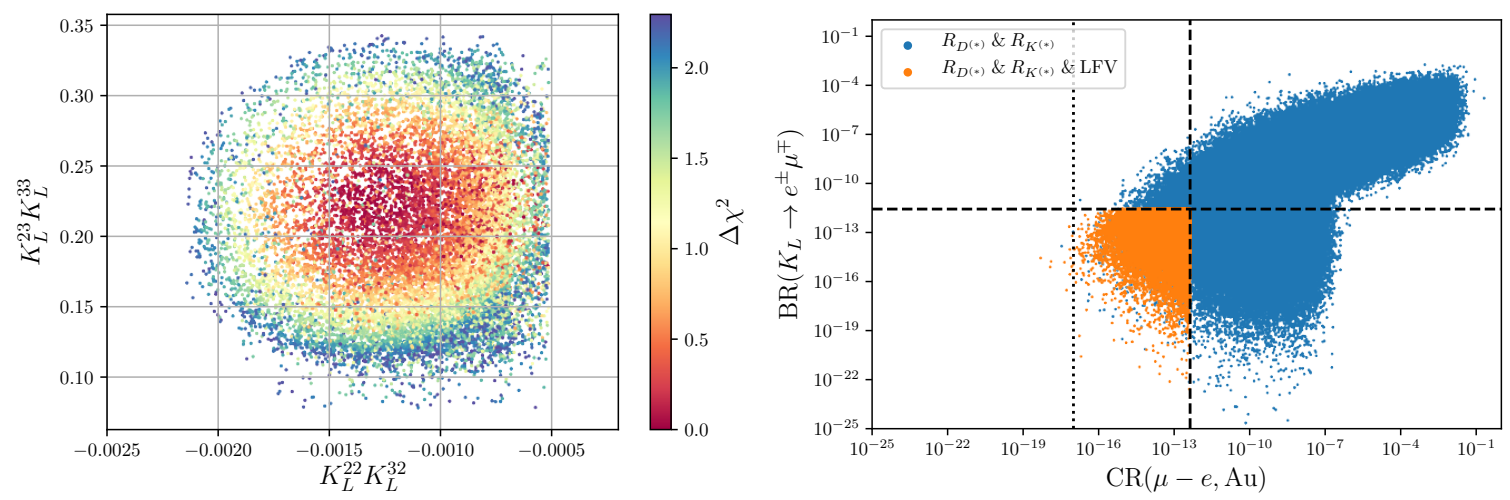

Figure 6. On the left, $\Delta \chi^{2}$ distribution for the fit to $R_{K^{(*)}}$ and $R_{D^{(*)}}$ data $(1 \sigma)$ in the plane of the $\left(K_{L}\right)_{i j}$ couplings. All points comply with the different (flavour) constraints. On the right, regions in the plane spanned by $\mathrm{CR}(\mu-e, \mathrm{~N})$ and $\mathrm{BR}\left(K_{L} \rightarrow \mu^{ \pm} e^{\mp}\right)$, accommodating both $R_{K^{(*)}}$ and $R_{D^{(*)}}$ (blue) and those in addition complying with LFV constraints (yellow). Both panels correspond to a heavy sector composed of three isodoublet vector-like charged lepton states, and to having set $m_{V} \sim 1.5 \mathrm{TeV}$. The $\Delta \chi^{2}$ corresponds to the $1 \sigma$-region around the best fit point.

The left panel of figure 6 offers a global view of this case, showing the $\Delta \chi^{2}$ distribution for the fit to $R_{K^{(*)}}$ and $R_{D^{(*)}}$ data, in the plane spanned by $\left(K_{L}\right)_{i j}$ "muon and tau couplings" ( $\left.K_{22} K_{32}-K_{23} K_{33}\right)$, marginalising over the other couplings. The leptoquark mass is set to $m_{V} \sim 1.5 \mathrm{TeV}$. We stress that leading to this plot all couplings were determined by the underlying nonunitarity parametrisation (with all mixing angles randomly sampled); in particular, we have not set the leptoquark couplings to the first generation of quark and leptons to zero. The displayed points comply with all flavour bounds included in our study, as described in section 3.2.

The lowest $\Delta \chi^{2}$ region (dark red ellipsoid) suggests that the best fit scenario corresponds to new physics dominantly coupling to muons and taus. We stress that the patterns emerging from the $\Delta \chi^{2}$ distribution are not an artefact of some particular assumption imposed on the couplings, but rather the result of a very general scan over the full set of (mixing) parameters.

The right panel of figure 6 offers a projection of the viable points (displayed on the left panel) in the plane of the most constraining observables, $\mathrm{CR}(\mu-e, \mathrm{~N})$ and $\mathrm{BR}\left(K_{L} \rightarrow\right.$ $\left.\mu^{ \pm} e^{\mp}\right)$. It is interesting to notice that, to a very good approximation, most of the currently phenomenologically viable points lie within future reach of the upcoming muon-electron conversion dedicated facilities (COMET and Mu2e).

In the near future, and should the $B$-meson decay anomalies be confirmed, an explanation in terms of such a minimal leptoquark framework could be probed via its impact for cLFV observables, in particular $\mu-e$ conversion in nuclei. Although the cLFV bounds could be evaded by increasing the mass of the vector leptoquark, this would however prevent a viable explanation of the $B$-meson decay anomalies, especially of $R_{D^{(*)}}$. 


\section{Concluding remarks}

In this study we have considered a minimal SM extension via one vector leptoquark $V_{1}$ and $n$ generations of heavy vector-like charged leptons, as a candidate framework to explain the current $B$-meson anomalies, $R_{K^{(*)}}$ and $R_{D^{(*)}}$.

Minimal extensions by a single $V_{1}$ leptoquark are in general disfavoured due to the strong cLFV constraints on the (unitary) quark-lepton- $V_{1}$ couplings. Here we have suggested that the pattern of mixings required to simultaneously address $R_{K^{(*)}}$ and $R_{D^{(*)}}$ with a single $V_{1}$ could be interpreted within a framework of nonunitary $V_{1} \ell q$ couplings: the mixings of the SM charged leptons with the additional vector-like heavy leptons can lead to effectively nonunitary $V_{1} \ell q$ couplings, offering the required amount of LFUV to account for both anomalies.

As we have argued, the most minimal nonunitary scenario (i.e. $n=1$ ) consistent with both $R_{K^{(*)}}$ and $R_{D^{(*)}}$, is ruled out as it leads to excessive contributions to cLFV observables such as muon-electron conversion in nuclei. We have thus considered three families of vector-like heavy leptons, and we have carried out a detailed analysis of the impact for an extensive array of flavour violation and EW precision observables. Our findings revealed that the $\mathrm{SU}(2)_{L}$ charges of the heavy charged leptons are of paramount importance for the model's viability: for isosinglet heavy leptons, the mass of the leptoquark must be sufficiently large to avoid excessive contributions to $Z$ decays, which then prevents an explanation of $R_{D^{(*)}}$. This is expected to happen for heavy leptons of any $\mathrm{SU}(2)_{L}$ representation except for isodoublets. In this particular case, the $Z \ell \ell$ couplings remain universal, and we have shown that the nonunitarity in the couplings allows to successfully explain both sets of anomalies, while complying with all considered current bounds.

The nonunitary framework here considered in fact provides one of the most economical options to simultaneously address $R_{K^{(*)}}$ and $R_{D^{(*)}}$ data via a single vector leptoquark. Although our results arise from a phenomenological analysis, our findings can be easily related to well-motivated UV complete models, in which the nonunitarity framework allows to obtain the required flavour structure without further enlarging the gauge group. Interestingly, this is the case of the Pati-Salam model [98-107], in which the existence of a vector leptoquark with a mass around the $\mathrm{TeV}$ scale (as required for a successful simultaneous explanation of both the neutral and charged current anomalies) naturally motivates the existence of such vector-like heavy leptons.

Finally, it is worth stressing that in view of the important progress expected in the near future, cLFV searches can play a crucial role in falsifying these minimal leptoquark frameworks, in view of their strong impact for cLFV observables, in particular $\mu-e$ conversion in nuclei. As we have discussed, should the $B$-meson decay anomalies be verified, and should this be a valid framework to explain them, then one should expect the observation of cLFV signals, in particular from $\mu-e$ conversion in Aluminium targets, either at COMET or Mu2e. 


\section{Acknowledgments}

We acknowledge support within the framework of the European Union's Horizon 2020 research and innovation programme under the Marie Sklodowska-Curie grant agreements No 690575 and No 674896.

\section{A Constraints from flavour violating rare meson decays and neutral me- son mixing}

New physics frameworks including leptoquarks give rise to important contributions to observables in the meson sector. These include flavour conserving and flavour violating leptonic and semileptonic decays ${ }^{9}$ (including final state neutrinos), as well as neutral meson mixings. In this appendix we discuss the different leptoquark contributions to leptonic and semi-leptonic meson decays (arising at tree-level) and to modes with final state neutrinos (at one-loop level). We recall that the SM predictions and current experimental limits are summarised in table 1.

Note that the matching of the model's parameters with the Wilson coefficients is performed at the scale of the leptoquark mass, and then these are run down to the $b$-quark mass scale. In our analysis, the running from the scale of leptoquark mass to the $b$-quark mass scale, and to any other relevant process (observable) scale, is taken into account using the wilson package [121] in association with the flavio package [122].

\section{A.1 Exclusive $d_{j} \rightarrow d_{i} \ell^{-} \ell^{\prime+}$ decays}

The effective Hamiltonian for $d_{j} \rightarrow d_{i} \ell^{-} \ell^{\prime+}$ transitions, including the LFV operators, can be cast as $[158-163]$

$$
\begin{aligned}
& \mathcal{H}_{\mathrm{eff}}=-\frac{4 G_{F}}{\sqrt{2}} V_{3 j} V_{3 i}^{*}\left[\sum_{\substack{k=7,9 \\
10, S, P}}\left(C_{k}(\mu) \mathcal{O}_{k}(\mu)+C_{k}^{\prime}(\mu) \mathcal{O}_{k}^{\prime}(\mu)\right)+C_{T}(\mu) \mathcal{O}_{T}(\mu)+C_{T_{5}}(\mu) \mathcal{O}_{T_{5}}(\mu)\right] \\
& .
\end{aligned}
$$

\footnotetext{
${ }^{9}$ We do not consider the $B_{s} \rightarrow \mu \mu$ decay explicitly, since it is already included in deriving the global ranges for the new physics contributions to $b \rightarrow$ sll anomalies.
} 
In the above, we recall that $V_{i j}$ denotes the CKM matrix; the operators are defined as

$$
\begin{aligned}
\mathcal{O}_{7}^{i j} & =\frac{e m_{d_{j}}}{(4 \pi)^{2}}\left(\bar{d}_{i} \sigma_{\mu \nu} P_{R} d_{j}\right) F^{\mu \nu}, \\
\mathcal{O}_{9}^{i j ; \ell \ell^{\prime}} & =\frac{e^{2}}{(4 \pi)^{2}}\left(\bar{d}_{i} \gamma^{\mu} P_{L} d_{j}\right)\left(\bar{\ell} \gamma_{\mu} \ell^{\prime}\right), \\
\mathcal{O}_{10}^{i j ; \ell \ell^{\prime}} & =\frac{e^{2}}{(4 \pi)^{2}}\left(\bar{d}_{i} \gamma^{\mu} P_{L} d_{j}\right)\left(\bar{\ell} \gamma_{\mu} \gamma_{5} \ell^{\prime}\right), \\
\mathcal{O}_{S}^{i j ; \ell \ell^{\prime}} & =\frac{e^{2}}{(4 \pi)^{2}}\left(\bar{d}_{i} P_{R} d_{j}\right)\left(\bar{\ell} \ell^{\prime}\right), \\
\mathcal{O}_{P}^{i j ; \ell \ell^{\prime}} & =\frac{e^{2}}{(4 \pi)^{2}}\left(\bar{d}_{i} P_{R} d_{j}\right)\left(\bar{\ell} \gamma_{5} \ell^{\prime}\right), \\
\mathcal{O}_{T}^{i j ; \ell \ell^{\prime}} & =\frac{e^{2}}{(4 \pi)^{2}}\left(\bar{d}_{i} \sigma_{\mu \nu} d_{j}\right)\left(\bar{\ell} \sigma^{\mu \nu} \ell^{\prime}\right), \\
\mathcal{O}_{T 5}^{i j ; \ell \ell^{\prime}} & =\frac{e^{2}}{(4 \pi)^{2}}\left(\bar{d}_{i} \sigma_{\mu \nu} d_{j}\right)\left(\bar{\ell} \sigma^{\mu \nu} \gamma_{5} \ell^{\prime}\right),
\end{aligned}
$$

with the primed operators $\mathcal{O}_{7,9,10, S, P}^{\prime}$ following from the replacement $P_{L} \leftrightarrow P_{R}$.

Regarding lepton flavour violating processes, only the operators $\mathcal{O}_{9,10, S, P}^{(\prime)}$ play a relevant role: for vector leptoquarks, the complete set of associated Wilson coefficients (not present in the SM) is given by [127]

$$
\begin{aligned}
C_{9,10}^{i j ; \ell \ell^{\prime}} & =\mp \frac{\pi}{\sqrt{2} G_{F} \alpha_{\mathrm{em}} V_{3 j} V_{3 i}^{*} m_{V}^{2}}\left(K_{L}^{i \ell^{\prime}} K_{L}^{j \ell *}\right) \\
C_{9,10}^{\prime i j ; \ell \ell^{\prime}} & =-\frac{\pi}{\sqrt{2} G_{F} \alpha_{\mathrm{em}} V_{3 j} V_{3 i}^{*} m_{V}^{2}}\left(K_{R}^{i \ell^{\prime}} K_{R}^{j \ell *}\right) \\
C_{S, P}^{i j ; \ell \ell^{\prime}} & = \pm \frac{\pi}{\sqrt{2} G_{F} \alpha_{\mathrm{em}} V_{3 j} V_{3 i}^{*} m_{V}^{2}}\left(K_{L}^{i \ell^{\prime}} K_{R}^{j \ell *}\right) \\
C_{S, P}^{\prime i j ; \ell \ell^{\prime}} & =\frac{\pi}{\sqrt{2} G_{F} \alpha_{\mathrm{em}} V_{3 j} V_{3 i}^{*} m_{V}^{2}}\left(K_{R}^{i \ell^{\prime}} K_{L}^{j \ell *}\right),
\end{aligned}
$$

where $K_{L}^{i \ell}\left(K_{R}^{i \ell}\right)$ denoting the left-handed (right-handed) leptoquark couplings. Here, and for completeness, we include the $K_{R}$ mixing matrix, although we do not take it into account in our analysis.

\section{A.2 $\quad P \rightarrow \ell^{-} \ell^{\prime+}$ decays}

Leptonic decays of pseudoscalar mesons lead to important constraints on the (vector) leptoquark couplings. Here, we summarise the computation of the $P \rightarrow \ell^{-} \ell^{\prime+}$ rates, following the derivation of [164]. With the standard decomposition of the hadronic matrix element

$$
\left\langle 0\left|\bar{d}_{j} \gamma_{\mu} \gamma_{5} d_{i}\right| P(p)\right\rangle=i p_{\mu} f_{P}
$$


in which $f_{P}$ denotes the $P$ meson decay constant, the branching fraction ${ }^{10}$ is given by

$$
\begin{aligned}
\operatorname{BR}(P & \left.\rightarrow \ell^{-} \ell^{\prime+}\right)=\frac{\tau_{P}}{64 \pi^{3}} \frac{\alpha^{2} G_{F}^{2}}{m_{P}^{3}} f_{P}^{2}\left|V_{3 j} V_{3 i}^{*}\right|^{2} \lambda^{\frac{1}{2}}\left(m_{P}, m_{\ell}, m_{\ell^{\prime}}\right) \\
& \times\left\{\left(m_{P}^{2}-\left(m_{\ell}+m_{\ell^{\prime}}\right)^{2}\right)\left|\left(C_{9}-C_{9}^{\prime}\right)\left(m_{\ell}-m_{\ell^{\prime}}\right)+\left(C_{S}-C_{S}^{\prime}\right) \frac{m_{P}^{2}}{m_{d_{j}}+m_{d_{i}}}\right|^{2}\right. \\
& \left.+\left(m_{P}^{2}-\left(m_{\ell}-m_{\ell^{\prime}}\right)^{2}\right)\left|\left(C_{10}-C_{10}^{\prime}\right)\left(m_{\ell}+m_{\ell^{\prime}}\right)+\left(C_{P}-C_{P}^{\prime}\right) \frac{m_{P}^{2}}{m_{d_{j}}+m_{d_{i}}}\right|^{2}\right\},
\end{aligned}
$$

where the Källén-function is defined as

$$
\lambda(a, b, c)=\left(a^{2}-(b-c)^{2}\right)\left(a^{2}-(b+c)^{2}\right) .
$$

Since this FCNC transition is generated at tree-level from $V_{1}$ interactions, the modes leading to different final state lepton charge assignments must both be included and treated separately. Leptonic pseudoscalar meson decays thus provide extensive (and very tight) constraints on the leptoquark couplings.

\section{A.3 $\quad P \rightarrow P^{\prime} \ell^{-} \ell^{\prime+}$ decays}

Again working in the standard basis, the hadronic matrix elements are parametrised as

$$
\begin{aligned}
\left\langle\bar{P}^{\prime}(k)\left|\bar{d}_{i} \gamma_{\mu} d_{j}\right| \bar{P}(p)\right\rangle & =\left[(p+k)_{\mu}-\frac{m_{P}^{2}-m_{P^{\prime}}^{2}}{q^{2}} q_{\mu}\right] f_{+}\left(q^{2}\right)+\frac{m_{P}^{2}-m_{P^{\prime}}^{2}}{q^{2}} q_{\mu} f_{0}\left(q^{2}\right), \\
\left\langle\bar{P}^{\prime}(k)\left|\bar{d}_{i} \sigma_{\mu \nu} d_{j}\right| \bar{P}(p)\right\rangle & =-i\left(p_{\mu} k_{\nu}-p_{\nu} k_{\mu}\right) \frac{2}{m_{P}+m_{P^{\prime}}} f_{T}\left(q^{2}, \mu\right) .
\end{aligned}
$$

In the above, the hadronic form factors $f_{+, 0, T}\left(q^{2}\right)$ depend on the momentum transfer, which lies in the range $\left(m_{\ell}+m_{\ell^{\prime}}\right)^{2} \leq q^{2} \leq\left(m_{P}-m_{P^{\prime}}\right)^{2}$. In our evaluation of the form factors we closely follow the results of [165]; since we focus on decays with heavy-to-light meson transitions, we further assume the scale to be $\mu=m_{d_{j}}$, i.e. $\mu=m_{b}$. With the quantities

$$
\begin{aligned}
\varphi_{7}\left(q^{2}\right)= & \frac{2 m_{d_{j}}\left|f_{T}\left(q^{2}\right)\right|^{2}}{\left(m_{P}+m_{P^{\prime}}\right)^{2}} \lambda\left(m_{P}, m_{P^{\prime}}, \sqrt{q^{2}}\right)\left[1-\frac{\left(m_{\ell}-m_{\ell^{\prime}}\right)^{2}}{q^{2}}-\frac{\lambda\left(\sqrt{q^{2}}, m_{\ell}, m_{\ell^{\prime}}\right)}{3 q^{4}}\right], \\
\varphi_{9(10)}\left(q^{2}\right)= & \frac{1}{2}\left|f_{0}\left(q^{2}\right)\right|^{2}\left(m_{\ell} \mp m_{\ell^{\prime}}\right)^{2} \frac{\left(m_{P}^{2}-m_{P^{\prime}}^{2}\right)^{2}}{q^{2}}\left[1-\frac{\left(m_{\ell} \pm m_{\ell^{\prime}}\right)^{2}}{q^{2}}\right] \\
& +\frac{1}{2}\left|f_{+}\left(q^{2}\right)\right|^{2} \lambda\left(m_{P}, m_{P^{\prime}}, \sqrt{q^{2}}\right)\left[1-\frac{\left(m_{\ell} \mp m_{\ell^{\prime}}\right)^{2}}{q^{2}}-\frac{\lambda\left(\sqrt{q^{2}}, m_{\ell}, m_{\ell^{\prime}}\right)}{3 q^{4}}\right], \\
\varphi_{79}\left(q^{2}\right)= & \frac{2 m_{d_{j}} f_{+}\left(q^{2}\right) f_{T}\left(q^{2}\right)}{m_{P}+m_{P^{\prime}}} \lambda\left(m_{P}, m_{P^{\prime}}, \sqrt{q^{2}}\right)\left[1-\frac{\left(m_{\ell}-m_{\ell^{\prime}}\right)^{2}}{q^{2}}-\frac{\lambda\left(\sqrt{q^{2}}, m_{\ell}, m_{\ell^{\prime}}\right)}{3 q^{4}}\right], \\
\varphi_{S(P)}\left(q^{2}\right)= & \frac{q^{2}\left|f_{0}\left(q^{2}\right)\right|^{2}}{2\left(m_{d_{j}}-m_{d_{i}}\right)^{2}}\left(m_{P}^{2}-m_{P^{\prime}}^{2}\right)^{2}\left[1-\frac{\left(m_{\ell} \pm m_{\ell^{\prime}}\right)^{2}}{q^{2}}\right], \\
\varphi_{10 P(9 S)}\left(q^{2}\right)= & \frac{\left|f_{0}\left(q^{2}\right)\right|^{2}}{m_{d_{j}}-m_{d_{i}}}\left(m_{\ell} \pm m_{\ell^{\prime}}\right)\left(m_{P}^{2}-m_{P^{\prime}}^{2}\right)^{2}\left[1-\frac{\left(m_{\ell} \mp m_{\ell^{\prime}}\right)^{2}}{q^{2}}\right],
\end{aligned}
$$

\footnotetext{
${ }^{10}$ Notice that for the lepton flavour conserving decay $B_{s} \rightarrow \mu \mu$, the SM contribution and the renormalisation group running (as well as mixing of the SM operators) must be taken into account.
} 
and the normalisation factor

$$
\left|\mathcal{N}_{P^{\prime}}\left(q^{2}\right)\right|^{2}=\tau_{P} \frac{\alpha^{2} G_{F}^{2}\left|V_{3 j} V_{3 i}^{*}\right|^{2}}{512 \pi^{5} m_{P}^{3}} \frac{\lambda^{\frac{1}{2}}\left(\sqrt{q^{2}}, m_{\ell}, m_{\ell^{\prime}}\right)}{q^{2}} \lambda^{\frac{1}{2}}\left(\sqrt{q^{2}}, m_{P}, m_{P^{\prime}}\right),
$$

the differential branching fraction can be cast as

$$
\begin{aligned}
& \frac{d \operatorname{BR}\left(P \rightarrow P^{\prime} \ell^{-} \ell^{\prime+}\right)}{d q^{2}}=\left|\mathcal{N}_{P^{\prime}}\left(q^{2}\right)\right|^{2} \\
& \times\left\{\varphi_{7}\left(q^{2}\right)\left|C_{7}+C_{7}^{\prime}\right|^{2}+\varphi_{9}\left(q^{2}\right)\left|C_{9}+C_{9}^{\prime}\right|^{2}+\varphi_{10}\left(q^{2}\right)\left|C_{10}+C_{10}^{\prime}\right|^{2}\right. \\
& \quad+\varphi_{S}\left(q^{2}\right)\left|C_{S}+C_{S}^{\prime}\right|^{2}+\varphi_{P}\left(q^{2}\right)\left|C_{P}+C_{P}^{\prime}\right|^{2}+\varphi_{79}\left(q^{2}\right) \operatorname{Re}\left[\left(C_{7}+C_{7}^{\prime}\right)\left(C_{9}+C_{9}^{\prime}\right)^{*}\right] \\
& \left.\quad+\varphi_{9 S}\left(q^{2}\right) \operatorname{Re}\left[\left(C_{9}+C_{9}^{\prime}\right)\left(C_{S}+C_{S}^{\prime}\right)^{*}\right]+\varphi_{10 P}\left(q^{2}\right) \operatorname{Re}\left[\left(C_{10}+C_{10}^{\prime}\right)\left(C_{P}+C_{P}^{\prime}\right)^{*}\right]\right\} .
\end{aligned}
$$

\section{A.4 Loop effects in neutrino modes}

Both $s \rightarrow d \nu \nu$ and $b \rightarrow s \nu \nu$ transitions are known to provide some of the most important constraints on NP scenarios aiming at addressing the anomalies in $R_{K^{(*)}}$ and $R_{D^{(*)}}$ data. Following the convention of [166], at the quark level the $|\Delta S|=1$ rare decays $K^{+}\left(K_{L}\right) \rightarrow$ $\pi^{+}\left(\pi^{0}\right) \nu_{\ell} \bar{\nu}_{\ell^{\prime}}$ and $B \rightarrow K^{(*)} \nu_{\ell} \bar{\nu}_{\ell^{\prime}}$ can be described by the following short-distance effective Hamiltonian for $d_{j} \rightarrow d_{i} \nu_{\ell} \bar{\nu}_{\ell^{\prime}}$ transitions [167, 168]

$$
\begin{aligned}
-\mathcal{H}_{\mathrm{eff}}=\frac{4 G_{F}}{\sqrt{2}} V_{3 i}^{*} V_{3 j} \frac{\alpha_{e}}{2 \pi}[ & C_{L, i j}^{\ell \ell^{\prime}}\left(\bar{d}_{i} \gamma_{\mu} P_{L} d_{j}\right)\left(\bar{\nu}_{\ell} \gamma^{\mu} P_{L} \nu_{\ell^{\prime}}\right) \\
& \left.+C_{R, i j}^{\ell \ell^{\prime}}\left(\bar{d}_{i} \gamma_{\mu} P_{R} d_{j}\right)\left(\bar{\nu}_{\ell} \gamma^{\mu} P_{L} \nu_{\ell^{\prime}}\right)\right]+ \text { H.c. },
\end{aligned}
$$

in which $i, j$ denote the down-type flavour content of the final and initial state meson, respectively. For vector leptoquarks, the contributions are generated at one loop-level and are a priori divergent; consequently, the calculation should be carried in a given gaugeembedding, including the corresponding would-be Goldstone modes. Here we follow the computation of [128], and the coefficient for $d_{a} \rightarrow d_{f} \bar{\nu}_{i} \nu_{j}$ is thus given by

$$
\begin{aligned}
C_{L, f a}^{i j}=\sum_{k, l}-\frac{M_{W}^{2}}{2 e^{2} V_{3 a} V_{3 f}^{*} m_{V}^{2}}( & 6 K_{L}^{f j} K_{L}^{a i *} \ln \left(\frac{M_{W}^{2}}{m_{V}^{2}}\right)+V_{3 f}^{*} V_{3 k} K_{L}^{k j} V_{3 a} V_{3 l}^{*} K_{L}^{l i *} \frac{m_{t}^{2}}{M_{W}^{2}}(\mathrm{~A} .13) \\
& \left.+3\left(V_{3 a} V_{3 k}^{*} K_{L}^{k i *} K_{L}^{f j}+V_{3 f}^{*} V_{3 k} K_{L}^{k j} K_{L}^{a i *}\right) \frac{m_{t}^{2} \ln \left(\frac{m_{t}^{2}}{M_{W}^{2}}\right)}{m_{t}^{2}-M_{W}^{2}}\right),
\end{aligned}
$$

where $M_{W}$ is the mass of the $W$ boson and $m_{t}$ the mass of the top quark. The branching fractions for the neutral and charged kaon decay modes are given by [129, 169]

$$
\begin{aligned}
\operatorname{BR}\left(K^{ \pm} \rightarrow \pi^{ \pm} \nu \bar{\nu}\right)= & \frac{1}{3}\left(1+\Delta_{E M}\right) \eta_{ \pm} \\
& \times \sum_{f, i=1}^{3}\left\{\left[\frac{\operatorname{Im}\left(\lambda_{t} \tilde{X}_{L}^{f i}\right)}{\lambda^{5}}\right]^{2}+\left[\frac{\operatorname{Re}\left(\lambda_{c}\right)}{\lambda} P_{c} \delta_{f i}+\frac{\operatorname{Re}\left(\lambda_{t} \tilde{X}_{L}^{f i}\right)}{\lambda^{5}}\right]^{2}\right\} \\
\operatorname{BR}\left(K_{L} \rightarrow \pi \nu \bar{\nu}\right)= & \frac{1}{3} \eta_{L} \sum_{f, i=1}^{3}\left[\frac{\operatorname{Im}\left(\lambda_{t} \tilde{X}_{L}^{f i}\right)}{\lambda^{5}}\right]^{2}
\end{aligned}
$$


with

$$
\begin{array}{rlrl}
\tilde{X}_{L}^{f i} & =X_{L}^{\mathrm{SM}, f i}-s_{W}^{2} C_{L, s d}^{f i}, & P_{c}=0.404 \pm 0.024 \\
\eta_{ \pm} & =(5.173 \pm 0.025) \times 10^{-11}\left[\frac{\lambda}{0.225}\right]^{8}, \\
\eta_{L} & =(2.231 \pm 0.013) \times 10^{-10}\left[\frac{\lambda}{0.225}\right]^{8}, \\
\Delta_{E M} & =-0.003, & X_{L}^{\mathrm{SM}, f i}=(1.481 \pm 0.005 \pm 0.008) \delta_{f i}
\end{array}
$$

In this convention, $\lambda$ is one of the Wolfenstein parameters related to the Cabibbo angle, and $\lambda_{c}=V_{c s}^{*} V_{c d}$ and $\lambda_{t}=V_{t s}^{*} V_{t d}$.

The $B \rightarrow K^{(*)} \nu \bar{\nu}$ decay width has been derived in [166], leading to $C_{L, s b}^{\mathrm{SM}, f i} \approx-1.47 / s_{W}^{2} \delta_{f i}$. Finally, it is convenient to express the BRs normalised to the SM predictions,

$$
R_{K^{(*)}}^{\nu \bar{\nu}}=\frac{1}{3} \sum_{f, i=1}^{3} \frac{\left|C_{L, s b}^{f i}\right|^{2}}{\left|C_{L, s b}^{\mathrm{SM}, f i}\right|^{2}} .
$$

\section{A.5 Loop effects in neutral meson mixing}

In this scenario a contribution to $|\Delta F|=2$ amplitudes is generated at one-loop level. Contributions to neutral meson mixings, $P-\bar{P}$ with $P=B_{s}^{0}, B_{d}^{0}, K^{0}$, arise both from SM box diagrams involving top quarks and $W$ 's, and from NP box diagrams involving leptons and vector leptoquarks. These contributions can be described in terms of the following effective Hamiltonian for $|\Delta F|=2$ transitions

$$
\mathcal{H}_{\text {eff }}^{P}=\left(C_{P}^{\mathrm{SM}}+C_{P}^{\mathrm{NP}}\right)\left(\bar{d}_{i} \gamma^{\mu} P_{L} d_{j}\right)\left(\bar{d}_{i} \gamma_{\mu} P_{L} d_{j}\right)+\text { H.c. },
$$

with $\{i, j\}$ respectively denoting $\{b, s\},\{b, d\}$ or $\{d, s\}$ for $P=B_{s}^{0}, B_{d}^{0}$ or $K^{0}$ mesons. The $|\Delta F|=2$ transitions are sensitive to the mass scale of the heavy vector-like fermions, and the widths scale proportionally to $m_{V}^{2}$ (similarly to the SM contribution, itself proportional to $m_{t}^{2}$ ). A complete evaluation of the contributions must further include the effects of the (physical) Higgs fields; therefore the computation of these observables requires specifying a particular UV completion. Nevertheless, it is possible to draw preliminary conclusions on the mass scales of the vector leptoquarks and heavy leptonic states (here denoted by $M$ ) based on the new physics contribution to the diagrams involving $V_{1}$. For example, taking $P=B_{s}^{0}$, one obtains $[97,98]$

$$
C_{B_{s}}^{\mathrm{NP}}=-\frac{K_{L}^{2 \ell} K_{L}^{3 \ell *} K_{L}^{2 \ell^{\prime}} K_{L}^{3 \ell^{\prime} *}}{16 \pi^{2}}\left(\frac{D_{6}}{4 M^{4}}+D_{2}-\frac{2 D_{4}}{M^{2}}\right)
$$

Here $\ell, \ell^{\prime}=1, \ldots, 6$ are the six fermions with the quantum numbers of charged leptons $(6$ physical eigenstates arising from the mixings of the light SM and heavy vector-like charged leptons). The loop functions $D_{x} \equiv D_{x}\left(M, M, m_{s}, m_{t}\right)$ are given by

$$
D_{x}\left(m_{1}, m_{2}, m_{3}, m_{4}\right)=\frac{i}{16 \pi^{2}} \int \frac{d^{d} k}{(2 \pi)^{d}} \frac{\left(k^{2}\right)^{x / 2}}{\left(k^{2}-m_{1}^{2}\right)\left(k^{2}-m_{2}^{2}\right)\left(k^{2}-m_{3}^{2}\right)\left(k^{2}-m_{4}^{2}\right)} .
$$


The $|\Delta F|=2$ transitions thus lead to a (lower) bound on the heavy leptonic mass scales of around $500 \mathrm{GeV}$, while the vector leptoquark mass should lie above the $\mathrm{TeV}$ to keep new physics contributions to $\Delta M_{B_{s, d}}$ below $\mathcal{O}(10 \%)$, given the experimental constraints.

\section{B Constraints from cLFV decays}

Due to the LFUV couplings of the leptoquarks, sizeable contributions can be generated for cLFV observables, including radiative decays $\ell_{i} \rightarrow \ell_{j} \gamma$, three-body decays $\ell_{i} \rightarrow 3 \ell_{j}$, and neutrinoless $\mu-e$ conversion in nuclei, which then lead to important constraints.

As already mentioned in section 3 , although the radiative decays are generated at loop level, anapole operators can induce additional contributions to the Wilson coefficients (comparable to the tree-level contributions to neutrinoless $\mu-e$ conversion), and dipole operators can also be responsible to significant contributions to radiative decays and neutrinoless $\mu-e$ conversion.

Notice that the one-loop dipole and anapole contributions due to the exchange of vector bosons generically diverge, and a UV completion must be specified to obtain a convergent result in a gauge independent manner. We have computed the anapole and dipole contributions in the Feynman gauge, for which it is necessary to include the relevant contributions from the Goldstone modes. To consistently compute these contributions for vector leptoquarks, we make the minimal working assumption that the new state corresponds to a (non-abelian) $\mathrm{SU}(3)_{c}$ gauge extension, whose breaking gives rise to a would-be Goldstone boson degree of freedom, subsequently absorbed by the massive vector leptoquark. We thus include this Goldstone mode (degenerate in mass with $V_{1}$ ) to obtain the gauge invariant (finite) form factors for the dipole and anapole contributions.

\section{B.1 Radiative lepton decays $\ell_{i} \rightarrow \ell_{j} \gamma$}

The relevant terms of the effective Lagrangian for radiative lepton decays $\ell_{i} \rightarrow \ell_{j} \gamma$ can be written as

$$
\mathcal{L}_{\text {eff }}^{\ell_{i} \rightarrow \ell_{j} \gamma}=-\frac{4 G_{F}}{\sqrt{2}} \bar{\ell}_{j} \sigma^{\mu \nu} F_{\mu \nu}\left(C_{L}^{\ell_{i} \ell_{j}} P_{L}+C_{R}^{\ell_{i} \ell_{j}} P_{R}\right) \ell_{i}+\text { H.c. },
$$

in which $F_{\mu \nu}$ is the electromagnetic field strength tensor. The corresponding Wilson coefficients are related to the form factors $\sigma_{L(R)}^{\ell_{i} \ell_{j}}$ as

$$
C_{L(R)}^{\ell_{i} \ell_{j}}=\frac{e}{4 \sqrt{2} G_{F}} \sigma_{L(R)}^{\ell_{i} \ell_{j}},
$$

where the form factors $\sigma_{L(R)}^{\ell_{i} \ell_{j}}$ can be computed following the prescription of [170]. At the one-loop level, there are 10 distinct diagrams that must be included. The gauge-invariant amplitude can be decomposed as

$$
i \mathcal{M}_{\text {dipole }}=i e \epsilon_{\mu}^{*} M_{\text {dipole }}^{\mu},
$$

with

$$
M_{\text {dipole }}^{\mu}=\bar{\ell}_{j}\left[i \sigma^{\mu \nu} q_{\nu}\left(\sigma_{L} P_{L}+\sigma_{R} P_{R}\right)\right] \ell_{i}
$$


in which $\epsilon$ and $q$ denote the photon polarisation and its momentum. The $\ell_{i} \rightarrow \ell_{j} \gamma$ decay width is then given by

$$
\Gamma\left(\ell_{i} \rightarrow \ell_{j} \gamma\right)=\frac{\alpha\left(m_{\ell_{i}}^{2}-m_{\ell_{j}}^{2}\right)^{3}}{4 m_{\ell_{i}}^{3}}\left(\left|\sigma_{L}^{\ell_{i} \ell_{j}}\right|^{2}+\left|\sigma_{R}^{\ell_{i} \ell_{j}}\right|^{2}\right) .
$$

In the physical (mass) basis, the relevant part of the Lagrangian leading to the computation of $\sigma_{L, R}$ can be written as (cf.eq. (2.4))

$$
\mathcal{L}_{V_{1}}=\sum_{i, j}\left[V_{1}^{\mu} \bar{d}_{i} \gamma_{\mu}\left(K_{L}^{i j} P_{L}+K_{R}^{i j} P_{R}\right) \ell_{j}+\text { H.c. }\right]
$$

with $K_{L}\left(K_{R}\right)$ the left-handed (right-handed) coupling matrix. The Goldstone $(\varphi)$ interaction terms in the Lagrangian are then given by

$$
\mathcal{L}_{\text {Goldstone }}=\varphi \frac{i}{M} \sum_{i, j} \bar{d}_{i}\left[\left(K_{R}^{i j} m_{\ell_{j}}-K_{L}^{i j} m_{d_{i}}\right) P_{L}+\left(K_{L}^{i j} m_{\ell_{j}}-K_{R}^{i j} m_{d_{i}}\right) P_{R}\right] \ell_{j}+\text { H.c. }
$$

The form factors $\sigma_{L, R}$ can be cast in the following (compact) form:

$$
\begin{array}{r}
\sigma_{L}^{\ell_{i} \ell_{j}}=-\frac{i N_{c}}{16 \pi^{2} M^{2}} \sum_{k}\left\{\frac{2}{3}\left[\left(K_{R}^{k j *} K_{R}^{k i} m_{\ell_{i}}+K_{L}^{k j *} K_{L}^{k i} m_{\ell_{j}}\right) g\left(t_{k}\right)+K_{R}^{k j *} K_{L}^{k i} m_{d_{k}} j\left(t_{k}\right)\right]\right. \\
\left.-\frac{1}{3}\left[\left(K_{R}^{k j *} K_{R}^{k i} m_{\ell_{i}}+K_{L}^{k j *} K_{L}^{k i} m_{\ell_{j}}\right) f\left(t_{k}\right)+K_{R}^{k j *} K_{L}^{k i} m_{d_{k}} h\left(t_{k}\right)\right]\right\}, \\
\sigma_{R}^{\ell_{i} \ell_{j}}=-\frac{i N_{c}}{16 \pi^{2} M^{2}} \sum_{k}\left\{\frac{2}{3}\left[\left(K_{L}^{k j *} K_{L}^{k i} m_{\ell_{i}}+K_{R}^{k j *} K_{R}^{k i} m_{\ell_{j}}\right) g\left(t_{k}\right)+K_{L}^{k j *} K_{R}^{k i} m_{d_{k}} j\left(t_{k}\right)\right]\right. \\
\left.-\frac{1}{3}\left[\left(K_{L}^{k j *} K_{L}^{k i} m_{\ell_{i}}+K_{R}^{k j *} K_{R}^{k i} m_{\ell_{j}}\right) f\left(t_{k}\right)+K_{L}^{k j *} K_{R}^{k i} m_{d_{k}} h\left(t_{k}\right)\right]\right\},
\end{array}
$$

with $t_{k}=\frac{m_{d_{k}}^{2}}{m_{V}^{2}}$ and $N_{c}$ the number of colours of the internal fermion. The loop functions are given by

$$
\begin{aligned}
& f(t)=\frac{-5 t^{3}+9 t^{2}-30 t+8}{12(t-1)^{3}}+\frac{3 t^{2} \ln (t)}{2(t-1)^{4}}, \\
& g(t)=\frac{-4 t^{3}+45 t^{2}-33 t+10}{12(t-1)^{3}}-\frac{3 t^{3} \ln (t)}{2(t-1)^{4}}, \\
& h(t)=\frac{t^{2}+t+4}{2(t-1)^{2}}-\frac{3 t \ln (t)}{(t-1)^{3}}, \\
& j(t)=\frac{t^{2}-11 t+4}{2(t-1)^{2}}+\frac{3 t^{2} \ln (t)}{(t-1)^{3}} .
\end{aligned}
$$

\section{B.2 Three body decays $\ell \rightarrow \ell^{\prime} \ell^{\prime} \ell^{\prime}$}

At the loop level, three body decays can receive contributions from photon penguins (dipole and off-shell "anapole"), $Z$ penguins and box diagrams, arising from flavour violating interactions involving the vector leptoquark $V_{1}$ and quarks. The relevant low-energy effective 
Lagrangian inducing the four-fermion operators responsible for $\ell \rightarrow \ell^{\prime} \ell^{\prime} \ell^{\prime}$ decays can be written as $[171,172]$

$$
\begin{aligned}
\mathcal{L}_{\ell \rightarrow \ell^{\prime} \ell^{\prime} \ell^{\prime}}=-\frac{4 G_{F}}{\sqrt{2}} & {\left[g_{1}\left(\overline{\ell^{\prime}} P_{L} \ell\right)\left(\overline{\ell^{\prime}} P_{L} \ell^{\prime}\right)+g_{2}\left(\overline{\ell^{\prime}} P_{R} \ell\right)\left(\overline{\ell^{\prime}} P_{R} \ell^{\prime}\right)+\right.} \\
& +g_{3}\left(\overline{\ell^{\prime}} \gamma^{\mu} P_{R} \ell\right)\left(\bar{\ell}^{\prime} \gamma_{\mu} P_{R} \ell^{\prime}\right)+g_{4}\left(\overline{\ell^{\prime}} \gamma^{\mu} P_{L} \ell\right)\left(\bar{\ell}^{\prime} \gamma_{\mu} P_{L} \ell^{\prime}\right)+ \\
& \left.+g_{5}\left(\overline{\ell^{\prime}} \gamma^{\mu} P_{R} \ell\right)\left(\bar{\ell}^{\prime} \gamma_{\mu} P_{L} \ell^{\prime}\right)+g_{6}\left(\bar{\ell}^{\prime} \gamma^{\mu} P_{L} \ell\right)\left(\bar{\ell}^{\prime} \gamma_{\mu} P_{R} \ell^{\prime}\right)\right]+ \text { H.c. }
\end{aligned}
$$

to which the photonic dipole terms entering in $\mathcal{L}_{\text {eff }}^{\ell_{i} \rightarrow \ell_{j} \gamma}$, cf. eq. (B.1), must be added; the corresponding coefficients parametrised by $C_{L(R)}^{\ell_{i} \ell_{j}}$ have already been discussed in detail in the previous subsection. Neglecting Higgs-mediated exchanges, the off-shell anapole photon penguins, $Z$ penguins and box diagrams will give rise to non-vanishing contributions to the above $g_{3}, g_{4}, g_{5}$ and $g_{6}$ coefficients. Note that in the large $V_{1}$ mass limit, the offshell anapole photon-penguin diagrams scale proportionally to $|K|^{2} \ln \left(m_{q}^{2} / M^{2}\right) / M^{2}$, in contrast with the contributions from the $Z$-penguins and box diagrams, which are (naïvely) proportional to $|K|^{2} m_{q}^{2} / M^{4}$ and $|K|^{4} m_{q}^{2} / M^{4}$ respectively [173]. Therefore we only include in our computation the log-enhanced photonic anapole contributions, in addition to the dipole ones. Neglecting right-handed couplings of the leptoquark as before, the only nonvanishing coefficients (at one-loop) are $g_{4}=g_{6}$. The relevant amplitude for the anapole contribution can be written as

$$
i \mathcal{M}_{\text {anapole }}=i e \epsilon_{\mu}^{*} M_{\text {anapole }}^{\mu}
$$

where $M_{\text {anapole }}^{\mu}$ can be parametrised in terms of a form factor $F_{L}^{\gamma \ell \ell^{\prime}}$ as

$$
\mathcal{M}_{\text {anapole }}^{\mu}=\frac{1}{(4 \pi)^{2}} F_{L}^{\gamma \ell \ell^{\prime}} \bar{\ell}^{\prime}\left(\gamma^{\mu} q^{2}-\not q q^{\mu}\right) P_{L} \ell
$$

with $q$ the off-shell photon momentum. In this convention the $F_{L}^{\gamma \ell \ell^{\prime}}$ form factor is independent of $q^{2}$. After performing the calculation in the Feynman gauge, we obtain (in the limit of vanishing external lepton masses)

$$
F_{L}^{\gamma \ell \ell^{\prime}}=\frac{N_{c}}{m_{V}^{2}} \sum_{i} K_{L}^{i \ell} K_{L}^{i \ell^{\prime} *} f_{a}\left(x_{i}\right)
$$

in which $x_{i}=m_{d_{i}}^{2} / m_{V}^{2}$ and $N_{c}$ is the colour factor (corresponding to the coloured fields entering in the loop). Finally, the loop function $f_{a}(x)$ is given by

$$
f_{a}(x)=\frac{4-26 x+15 x^{2}+x^{3}}{12(1-x)^{3}}+\frac{4-16 x-15 x^{2}+20 x^{3}-2 x^{4}}{18(1-x)^{4}} \ln (x) .
$$

The leptoquark-induced contributions to the 4-fermion operators are given by

$$
g_{4}=g_{6}=-\frac{\sqrt{2}}{4 G_{F}} \frac{\alpha}{4 \pi} Q_{f} F_{L}^{\gamma \ell \ell^{\prime}} .
$$


In the case of the $\ell \rightarrow 3 \ell^{\prime}$ decays, $Q_{f}=Q_{\ell}^{\prime}$ denotes the charge of the fermion pair at the end of the off-shell photon (in units of $e$ ). As an example, for the case of $\mu \rightarrow 3 e$ decays, one obtains the following branching ratio $[171,172]$

$$
\begin{aligned}
\operatorname{BR}(\mu \rightarrow e e e)= & 2\left(\left|g_{3}\right|^{2}+\left|g_{4}\right|^{2}\right)+\left|g_{5}\right|^{2}+\left|g_{6}\right|^{2}+ \\
& +8 e \operatorname{Re}\left[C_{R}^{\mu e}\left(2 g_{4}^{*}+g_{6}^{*}\right)+C_{L}^{\mu e}\left(2 g_{3}^{*}+g_{5}^{*}\right)\right]+ \\
& +\frac{32 e^{2}}{m_{\mu}^{2}}\left\{\ln \frac{m_{\mu}^{2}}{m_{e}^{2}}-\frac{11}{4}\right\}\left(\left|C_{R}^{\mu e}\right|^{2}+\left|C_{L}^{\mu e}\right|^{2}\right) .
\end{aligned}
$$

Similar expressions can be easily inferred for the other cLFV 3-body decay channels.

\section{B.3 Neutrinoless $\mu-e$ conversion}

In terms of the relevant effective Wilson coefficients, the general contribution to the neutrinoless $\mu-e$ conversion rate is given by [127]

$$
\begin{aligned}
\Gamma_{\mu-e, \mathrm{~N}}=2 G_{F}^{2}(\mid & \frac{C_{R}^{\mu e *}}{m_{\mu}} D+\left(2 g_{L V}^{(u)}+g_{L V}^{(d)}\right) V^{(p)}+\left(g_{L V}^{(u)}+2 g_{L V}^{(d)}\right) V^{(n)} \\
& +\left(G_{S}^{(u, p)} g_{L S}^{(u)}+G_{S}^{(d, p)} g_{L S}^{(d)}+G_{S}^{(s, p)} g_{L S}^{(s)}\right) S^{(p)} \\
& \left.+\left.\left(G_{S}^{(u, n)} g_{L S}^{(u)}+G_{S}^{(d, n)} g_{L S}^{(d)}+G_{S}^{(s, n)} g_{L S}^{(s)}\right) S^{(n)}\right|^{2}+(L \leftrightarrow R)\right),
\end{aligned}
$$

in which the photonic dipole Wilson coefficients $C_{L(R)}^{\ell_{i} \ell_{j}}$ have been given in eq. (B.1); the other non-vanishing coefficients, induced by the tree-level leptoquark exchange or arising from the photonic anapole contributions, are given by

$$
\begin{aligned}
g_{L V}^{(d)} & =\frac{\sqrt{2}}{G_{F}}\left(\frac{1}{m_{V}^{2}} K_{L}^{d e} K_{L}^{d \mu *}+\frac{\alpha}{4 \pi} Q_{d} F_{L}^{\gamma \mu e}\right) \\
g_{L V}^{(u)} & =\frac{\sqrt{2}}{G_{F}}\left(\frac{\alpha}{4 \pi} Q_{u} F_{L}^{\gamma \mu e}\right) \\
g_{R V}^{(d)} & =\frac{\sqrt{2}}{G_{F}}\left(\frac{\alpha}{4 \pi} Q_{d} F_{L}^{\gamma \mu e}\right) \\
g_{R V}^{(u)} & =\frac{\sqrt{2}}{G_{F}}\left(\frac{\alpha}{4 \pi} Q_{u} F_{L}^{\gamma \mu e}\right),
\end{aligned}
$$

with $Q_{d}=-\frac{1}{3}$ and $Q_{u}=\frac{2}{3}$. The values for the overlap integrals $(D, V, S)$ are given in table 4 [174], and the scalar coefficients $G_{S}^{\left(d_{i}, N\right)}$ can be found in [175]. We again emphasise here that the off-shell anapole contributions, often neglected in the literature, can have a contribution comparable to the tree-level leptoquark exchange, and therefore should be included for a thorough estimation of the rate of $\mu-e$ conversion in nuclei.

\section{Electroweak precision observables}

As mentioned in the main body of the paper, the hypothetical heavy vector-like fermions can modify the couplings of SM fermions to gauge bosons ${ }^{11}$ leading to deviations from

\footnotetext{
${ }^{11}$ As before, parallels can be drawn with respect to the case in which heavy neutral leptons, with nonnegligible mixings to the SM neutrinos, are added to the SM field content.
} 


\begin{tabular}{|lccc|}
\hline Nucleus & $D\left[m_{\mu}^{5 / 2}\right]$ & $V^{(p)}\left[m_{\mu}^{5 / 2}\right]$ & $V^{(n)}\left[m_{\mu}^{5 / 2}\right]$ \\
\hline $\mathrm{Ti}_{22}^{48}$ & 0.0864 & 0.0396 & 0.0468 \\
$\mathrm{Au}_{79}^{197}$ & 0.189 & 0.0974 & 0.146 \\
\hline & $S^{(p)}\left[m_{\mu}^{5 / 2}\right]$ & $S^{(n)}\left[m_{\mu}^{5 / 2}\right]$ & $\Gamma_{\text {capture }}\left[10^{6} \mathrm{~s}^{-1}\right]$ \\
\hline $\mathrm{Ti}_{22}^{48}$ & 0.0368 & 0.0435 & 2.59 \\
$\mathrm{Au}_{79}^{197}$ & 0.0614 & 0.0918 & 13.07 \\
\hline
\end{tabular}

Table 4. Overlap integrals $D, V, S$ and $\Gamma_{\text {capture }}$ for Gold and Titanium nuclei, as reported in [174] (tables I and VIII).

the theoretical predictions of the SM, which are mostly in remarkable agreement with EW precision data.

\section{C.1 Couplings of the $Z$ boson and photon}

If the heavy vector-like fermion states are $\mathrm{SU}(2)_{L}$ singlets, mixings with the light $\mathrm{SU}(2)_{L}$ doublets can lead to modified couplings of the latter to the $Z$ boson $(\bar{f} f Z)$. For the case of charged leptons, the relevant couplings can be obtained from the kinetic terms,

$$
\mathcal{L}_{\text {kin }} \supset \bar{\ell}_{L a}^{0} i \not D_{a} \ell_{L a}^{0}+\bar{\ell}_{R a}^{0} i \not D_{a} \ell_{R a}^{0}=\bar{\ell}_{L j}\left(U_{L}^{\ell}\right)_{j a}^{\dagger} i \not D_{a}\left(U_{L}^{\ell}\right)_{a k} \ell_{L k}+\bar{\ell}_{R j}\left(U_{R}^{\ell}\right)_{j a}^{\dagger} i \not D_{a}\left(U_{R}^{\ell}\right)_{a k} \ell_{R k},
$$

where $\{j, k\}$ denotes the physical fields and $\{a, b\}=1 \ldots 6$ the interaction states (with $a \in\{1,2,3\}$ corresponding to SM fermions, and $a \in\{4,5,6\}$ to the new heavy vector-like states). The covariant derivative associated with the charges of a given state $a$ can thus be written

$$
D_{\mu, a}=\partial_{\mu}-i \frac{g}{\cos \theta_{W}}\left(T_{a}^{3}-\sin ^{2} \theta_{W} Q_{a}\right) Z_{\mu}-i e Q_{a} A_{\mu}
$$

where $T^{3}$ and $Q$ respectively denote the weak isospin and the electric charge. Since the electric charge is the same for all lepton states $\left(Q_{a}=-1\right)$, the couplings of the photon are not modified.

Let us now introduce the "effective" $Z$ boson couplings,

$$
\begin{aligned}
& \left(g_{L}^{Z \ell_{j} \ell_{k}}\right)_{\mathrm{eff}}=\sum_{a=1}^{6} \frac{g}{\cos \theta_{W}}\left(T_{L a}^{3}-\sin ^{2} \theta_{W} Q_{a}\right)\left(U_{L}^{\ell}\right)_{j a}^{\dagger}\left(U_{L}^{\ell}\right)_{a k}, \\
& \left(g_{R}^{Z \ell_{j} \ell_{k}}\right)_{\mathrm{eff}}=\sum_{a=1}^{6} \frac{g}{\cos \theta_{W}}\left(T_{R a}^{3}-\sin ^{2} \theta_{W} Q_{a}\right)\left(U_{R}^{\ell}\right)_{j a}^{\dagger}\left(U_{R}^{\ell}\right)_{a k},
\end{aligned}
$$

where $T_{L(R)}^{3}$ is the weak isospin of a left-handed (right-handed) lepton. Should the SM fermions and the heavy states belong to the same $\mathrm{SU}(2)_{L}$ representation, universality is trivially restored (by unitarity) for both $g_{L, R}^{Z \ell_{j} \ell_{k}}$ effective couplings, and one recovers the SM universal couplings. For heavy isodoublet vector-like states, one has

$$
\left(g_{L}^{Z \ell_{j} \ell_{k}}\right)_{\mathrm{eff}}=\frac{g}{\cos \theta_{W}}\left(-\frac{1}{2}+\sin ^{2} \theta_{W}\right) \delta_{j k},
$$


However, if the new fields transform differently (have distinct charges) under $\mathrm{SU}(2)_{L}$, the

$g_{L, R}^{Z \ell_{j} \ell_{k}}$ couplings are modified. In particular, in the presence of isosinglet heavy states, one finds

$\left(g_{L}^{Z \ell_{j} \ell_{k}}\right)_{\mathrm{eff}}=\frac{g}{\cos \theta_{W}}\left(-\frac{1}{2}+\sin ^{2} \theta_{W}\right) \delta_{j k}+\Delta g_{L}^{j k}, \quad$ with $\quad \Delta g_{L}^{j k}=\sum_{a=4}^{6} \frac{1}{2} \frac{g}{\cos \theta_{W}}\left(U_{L}^{\ell}\right)_{j a}^{\dagger}\left(U_{L}^{\ell}\right)_{a k}$.

Likewise, vector-like doublets also lead to the modification of the $g_{R}^{Z \ell_{j} \ell_{k}}$ couplings:

$$
\left(g_{R}^{Z \ell_{j} \ell_{k}}\right)_{\mathrm{eff}}=\frac{g}{\cos \theta_{W}} \sin ^{2} \theta_{W} \delta_{j k}+\Delta g_{R}^{j k}, \quad \text { with } \quad \Delta g_{R}^{j k}=\sum_{a=4}^{6}-\frac{1}{2} \frac{g}{\cos \theta_{W}}\left(U_{R}^{\ell}\right)_{j a}^{\dagger}\left(U_{R}^{\ell}\right)_{a k} .
$$

\section{C.2 Couplings of the $W$ boson}

The possible mixings with the heavy vector-like leptons can also modify the couplings to the $W$ boson. The charged current interaction terms can be written

$$
\begin{aligned}
\mathcal{L}^{\mathrm{cc}} & =\frac{g}{\sqrt{2}} W_{\mu} \bar{\nu}_{a}^{0} \gamma^{\mu} \ell_{L a}^{0}+\text { H.c. }, \\
& =\frac{g}{\sqrt{2}} W_{\mu} \bar{\nu}_{j} \gamma^{\mu}\left(U_{L}^{\nu \dagger}\right)_{j a}\left(U_{L}\right)_{a k} \ell_{L k}+\text { H.c. },
\end{aligned}
$$

so that the corresponding charged current couplings are then given by

$$
g_{L}^{W \nu_{j} \ell_{k}}=\frac{g}{\sqrt{2}}\left(U_{L}^{\nu \dagger}\right)_{j a}\left(U_{L}^{\ell}\right)_{a k}=\frac{g}{\sqrt{2}} U_{j k}^{\mathrm{P} \dagger},
$$

where $U^{\mathrm{P}}$ denotes the (generalised) PMNS mixing matrix. A priori, the branching ratios of $W \rightarrow \ell \nu$ can constrain the mixings of the heavy leptons (see, e.g. [176, 177]). However, these strongly depend on the neutrino mass generation mechanism (as well as on the structure of the Higgs sector), and in the present analysis we will not take them into account; we nevertheless mention that for a given Higgs sector, in the presence of additional isosinglet heavy neutrinos, the modified charged current vertex can impact several observables. Therefore, in addition to electroweak precision measurements of $\mathrm{BR}(W \rightarrow \ell \nu)$ [178], other decays or collider processes with one or two neutrinos in the final state, as for example $\tau$ decays, leptonic and semileptonic meson decays [179-181], production and decay of $W$ bosons to dilepton and two jets at the LHC [182, 183], can also lead to interesting constraints depending on the mass scales of the additional isosinglet neutral states.

\section{C.3 Constraining EWP observables}

Due to the tree-level modified $Z$-couplings (a consequence of the mixing of SM fermions with the heavy vector-like fermions), strong constraints from EWP observables are expected to arise from the observed lepton universality in charged leptonic $Z$-decays. 
At tree level, the decay width of a massive vector boson to fermions is given by [178]

$$
\begin{aligned}
& \Gamma\left(V \rightarrow f f^{\prime}\right)=\frac{\lambda^{1 / 2}\left(m_{V}, m_{f}, m_{f^{\prime}}\right)}{48 \pi m_{V}^{3}} \\
& \times\left[\left(\left|g_{L}^{f f^{\prime}}\right|^{2}+\left|g_{R}^{f f^{\prime}}\right|^{2}\right)\left(2 m_{V}^{2}-m_{f}^{2}-m_{f^{\prime}}^{2}-\frac{\left(m_{f}^{2}-m_{f^{\prime}}^{2}\right)^{2}}{m_{V}^{2}}\right)+12 m_{f} m_{f^{\prime}} \operatorname{Re}\left(g_{L}^{f f^{\prime}} g_{R}^{f f^{\prime} *}\right)\right],
\end{aligned}
$$

where $g_{L(R)}$ are the chiral couplings and the Källén function is defined in eq. (A.6). In the case of the $Z$-boson, the relevant couplings have been introduced in eq. (C.3). From eq. (C.9), and in view of the very good agreement between the SM predictions and experiment (cf. table 2), it is clear that any modification of the tree-level couplings of the $Z$-boson will be subject to very stringent constraints, which in turn translate into bounds on the mixing parameters responsible for the $\Delta g_{L(R)}$ terms (see eqs. (C.5), (C.6)). Using eq. (C.9), one can derive conservative constraints on $\Delta g_{L(R)}$ from the requirement of compatibility with the bounds of table 2 . As an example, the current experimental data on $\operatorname{BR}\left(Z \rightarrow e^{ \pm} \mu^{\mp}\right)$ and $\Gamma\left(\ell^{+} \ell^{-}\right)$leads to

$$
\begin{aligned}
\left|\Delta g_{L}^{e \mu}\right|^{2}+\left|\Delta g_{R}^{e \mu}\right|^{2} & \lesssim 1.55 \times 10^{-6}, \\
\left|\Delta g_{L}^{\ell \ell}\right| & \lesssim 5.6 \times 10^{-4}, \\
\left|\Delta g_{R}^{\ell \ell}\right| & \lesssim 3.5 \times 10^{-4} .
\end{aligned}
$$

The above constraints allow in turn to infer bounds on the elements of the matrix $A$ (see eqs. (2.7)-(2.11)). To illustrate this point, we consider the case of isosinglet heavy vector-like leptons (for which $\Delta g_{R}=0$ ): ${ }^{12}$ the limits of eq. (C.10) translate into

$$
\begin{aligned}
1-\left|\alpha_{11}\right| & \lesssim 4 \times 10^{-4}, \\
1-\left|\alpha_{22}\right| & \lesssim 3 \times 10^{-4}, \\
\left|\alpha_{21}\right| & \lesssim 4.6 \times 10^{-4} .
\end{aligned}
$$

\section{Details of the numerical analysis}

In what follows, we detail relevant aspects of the numerical analysis done in sections 2 and 3 , in particular concerning the global fits and the scans over the parameter space leading to the different plots. The global fits displayed in figures 2 and 3 are obtained using the "fastfit" method of the flavio package [122]. This is based on the approximation which assumes the likelihood to be of the form $\mathcal{L}=e^{-\chi^{2}(\vec{\xi}) / 2}$ where

$$
\chi^{2}(\vec{\xi})=\vec{\Delta}^{T} C^{-1}(\vec{\xi}=\hat{\vec{\xi}}) \vec{\Delta}, \quad \Delta_{i}=\left(x^{\exp }-x_{i}^{\mathrm{th}}(\vec{\theta})\right)
$$

\footnotetext{
${ }^{12}$ Note that in the presence of a nontrivial Higgs sector inducing mixings between right-handed SM charged leptons and vector-like doublets, one has non-vanishing contributions to $\Delta g_{R}$, which can lead to constraints on the right-handed mixing matrix, parametrised as done for $K_{L}$, see eq. (2.5), for a given UV complete framework. However, a detailed analysis of such a scenario is beyond the scope of our current work. Here, we only include the conservative limits for left-handed mixing elements, which are of foremost importance to our analysis.
} 
and $C(\vec{\xi})=C_{\exp }+C_{\mathrm{th}}(\vec{\xi})$ being the combined (theoretical and experimental) covariance matrix of the observables $x_{i}$ and the theoretical and experimental uncertainties are approximated as Gaussian. For a more detailed description of the statistical treatment, we refer the reader to ref. [184]. Likelihood contours are obtained by calculating the $\Delta \chi^{2}$ deviation around the best-fit point.

To obtain the various scatter plots, we have varied all mixing parameters of the parametrisation as stated in the captions. The mass of the vector leptoquark is varied in the ranges described in the captions of the figures (or set to a fixed value). The mixing angles were varied between $-\pi$ and $\pi$. To overcome the difficulties associated with presenting a parameter space spanned by 12 mixing angles in the nonunitary parametrisation, we perform a random scan using $\sim 10^{10} n$-tuples of random numbers and compute the predictions of the model for a large number of flavour violating observables, including those shown in tables 1 and 3. If any of the predictions for a certain parameter $n$-tuple exceeds the experimental bounds in a given set of constraints, the point is filtered in the appropriate category. Points complying with the imposed constraints are further processed to calculate their global likelihood regarding the $b \rightarrow$ sll and $b \rightarrow c \ell \nu$ observables using flavio [122]. Relevant effects due to renormalisation group running, in all the relevant processes (which we have used as constraints or for fitting procedures in our analysis) were computed with the wilson package [121] in association with the flavio package [122].

Open Access. This article is distributed under the terms of the Creative Commons Attribution License (CC-BY 4.0), which permits any use, distribution and reproduction in any medium, provided the original author(s) and source are credited.

\section{References}

[1] Particle Data Group collaboration, Review of particle physics, Phys. Rev. D 98 (2018) 030001 [INSPIRE].

[2] ALEPH, DELPHI, L3, OPAL, SLD, LEP Electroweak Working Group, SLD Electroweak Group and SLD Heavy Flavour Group collaborations, Precision electroweak measurements on the $Z$ resonance, Phys. Rept. 427 (2006) 257 [hep-ex/0509008] [INSPIRE].

[3] BABAR collaboration, Evidence for an excess of $\bar{B} \rightarrow D^{(*)} \tau^{-} \bar{\nu}_{\tau}$ decays, Phys. Rev. Lett. 109 (2012) 101802 [arXiv: 1205.5442] [INSPIRE].

[4] BABAR collaboration, Measurement of an excess of $\bar{B} \rightarrow D^{(*)} \tau^{-} \bar{\nu}_{\tau}$ decays and implications for charged Higgs bosons, Phys. Rev. D 88 (2013) 072012 [arXiv: 1303.0571] [INSPIRE].

[5] BeLLe collaboration, Measurement of the branching ratio of $\bar{B} \rightarrow D^{(*)} \tau^{-} \bar{\nu}_{\tau}$ relative to $\bar{B} \rightarrow D^{(*)} \ell^{-} \bar{\nu}_{\ell}$ decays with hadronic tagging at Belle, Phys. Rev. D 92 (2015) 072014 [arXiv: 1507.03233] [INSPIRE].

[6] BeLLe collaboration, Measurement of $B \rightarrow D(*) \tau \nu$ using full reconstruction tags, in Proceedings, $24^{\text {th }}$ International Symposium on Lepton-Photon Interactions at High Energy (LP09), Hamburg, Germany, 17-22 August 2009 [arXiv:0910.4301] [INSPIRE].

[7] Belle collaboration, Observation of $B^{+} \rightarrow \bar{D}^{* 0} \tau^{+} \nu_{\tau}$ and evidence for $B^{+} \rightarrow \bar{D}^{0} \tau^{+} \nu_{\tau}$ at Belle, Phys. Rev. D 82 (2010) 072005 [arXiv: 1005.2302] [inSPIRE]. 
[8] LHCb collaboration, Measurement of the ratio of branching fractions $\mathcal{B}\left(\bar{B}^{0} \rightarrow D^{*+} \tau^{-} \bar{\nu}_{\tau}\right) / \mathcal{B}\left(\bar{B}^{0} \rightarrow D^{*+} \mu^{-} \bar{\nu}_{\mu}\right)$, Phys. Rev. Lett. 115 (2015) 111803 [Erratum ibid. 115 (2015) 159901] [arXiv:1506.08614] [INSPIRE].

[9] BeLle collaboration, Measurement of the $\tau$ lepton polarization and $R\left(D^{*}\right)$ in the decay $\bar{B} \rightarrow D^{*} \tau^{-} \bar{\nu}_{\tau}$, Phys. Rev. Lett. 118 (2017) 211801 [arXiv:1612.00529] [INSPIRE].

[10] BeLle collaboration, Measurement of $\mathcal{R}(D)$ and $\mathcal{R}\left(D^{*}\right)$ with a semileptonic tagging method, arXiv:1904.08794 [INSPIRE].

[11] LHCb collaboration, Search for lepton-universality violation in $B^{+} \rightarrow K^{+} \ell^{+} \ell^{-}$decays, Phys. Rev. Lett. 122 (2019) 191801 [arXiv:1903.09252] [INSPIRE].

[12] LHCb collaboration, Test of lepton universality with $B^{0} \rightarrow K^{* 0} \ell^{+} \ell^{-}$decays, JHEP 08 (2017) 055 [arXiv: 1705. 05802] [inSPIRE].

[13] BeLLe collaboration, Test of lepton flavor universality in $B \rightarrow K^{*} \ell^{+} \ell^{-}$decays at Belle, arXiv: 1904.02440 [INSPIRE].

[14] LHCb collaboration, Angular analysis and differential branching fraction of the decay $B_{s}^{0} \rightarrow \phi \mu^{+} \mu^{-}$, JHEP 09 (2015) 179 [arXiv: 1506. 08777] [INSPIRE].

[15] BELLE collaboration, Lepton-flavor-dependent angular analysis of $B \rightarrow K^{*} \ell^{+} \ell^{-}$, Phys. Rev. Lett. 118 (2017) 111801 [arXiv:1612.05014] [INSPIRE].

[16] HFLAV collaboration, Averages of b-hadron, c-hadron and $\tau$-lepton properties as of summer 2016, Eur. Phys. J. C 77 (2017) 895 [arXiv: 1612.07233] [InSPIRE].

[17] Average of $R(D)$ and $R\left(D^{*+}\right)$ for Spring 2019 webpage, https://hflav-eos.web.cern.ch/hflav-eos/semi/spring19/html/RDsDsstar/RDRDs.html.

[18] D. Bigi and P. Gambino, Revisiting B $\rightarrow$ D $\ell$, Phys. Rev. D 94 (2016) 094008 [arXiv: 1606.08030] [INSPIRE].

[19] D. Bigi, P. Gambino and S. Schacht, $R\left(D^{*}\right),\left|V_{c b}\right|$ and the heavy quark symmetry relations between form factors, JHEP 11 (2017) 061 [arXiv:1707.09509] [INSPIRE].

[20] Z. Ligeti, M. Papucci and D.J. Robinson, New physics in the visible final states of $B \rightarrow D^{(*)} \tau \nu$, JHEP 01 (2017) 083 [arXiv: 1610.02045] [INSPIRE].

[21] A. Crivellin, J. Fuentes-Martin, A. Greljo and G. Isidori, Lepton flavor non-universality in B decays from dynamical Yukawas, Phys. Lett. B 766 (2017) 77 [arXiv:1611.02703] [INSPIRE].

[22] M. Bordone, G. Isidori and A. Pattori, On the Standard Model predictions for $R_{K}$ and $R_{K^{*}}$, Eur. Phys. J. C 76 (2016) 440 [arXiv: 1605.07633] [InSPIRE].

[23] B. Capdevila, A. Crivellin, S. Descotes-Genon, J. Matias and J. Virto, Patterns of new physics in $b \rightarrow s \ell^{+} \ell^{-}$transitions in the light of recent data, JHEP 01 (2018) 093 [arXiv: 1704.05340] [INSPIRE].

[24] W. Altmannshofer and D.M. Straub, New physics in $b \rightarrow s$ transitions after LHC run 1 , Eur. Phys. J. C 75 (2015) 382 [arXiv:1411.3161] [InSPIRE].

[25] A. Bharucha, D.M. Straub and R. Zwicky, $B \rightarrow V \ell^{+} \ell^{-}$in the Standard Model from light-cone sum rules, JHEP 08 (2016) 098 [arXiv: 1503.05534] [INSPIRE].

[26] M. Algueró et al., Emerging patterns of new physics with and without lepton flavour universal contributions, Eur. Phys. J. C 79 (2019) 714 [arXiv:1903.09578] [INSPIRE]. 
[27] J. Aebischer, W. Altmannshofer, D. Guadagnoli, M. Reboud, P. Stangl and D.M. Straub, B-decay discrepancies after Moriond 2019, arXiv:1903.10434 [INSPIRE].

[28] M. Ciuchini et al., New physics in $b \rightarrow s \ell^{+} \ell^{-}$confronts new data on lepton universality, Eur. Phys. J. C 79 (2019) 719 [arXiv: 1903.09632] [InSPIRE].

[29] A. Datta, J. Kumar and D. London, The $B$ anomalies and new physics in $b \rightarrow s e^{+} e^{-}$, Phys. Lett. B 797 (2019) 134858 [arXiv:1903.10086] [INSPIRE].

[30] A. Arbey, T. Hurth, F. Mahmoudi, D.M. Santos and S. Neshatpour, Update on the $b \rightarrow s$ anomalies, Phys. Rev. D 100 (2019) 015045 [arXiv: 1904.08399] [INSPIRE].

[31] R.-X. Shi, L.-S. Geng, B. Grinstein, S. Jäger and J. Martin Camalich, Revisiting the new-physics interpretation of the $b \rightarrow c \tau \nu$ data, arXiv:1905.08498 [INSPIRE].

[32] D. Bardhan and D. Ghosh, B-meson charged current anomalies: the post-Moriond 2019 status, Phys. Rev. D 100 (2019) 011701 [arXiv: 1904.10432] [InSPIRE].

[33] A.K. Alok, A. Dighe, S. Gangal and D. Kumar, Continuing search for new physics in

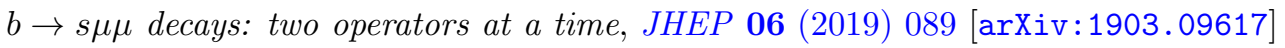
[INSPIRE].

[34] A.K. Alok, D. Kumar, J. Kumar, S. Kumbhakar and S.U. Sankar, New physics solutions for $R_{D}$ and $R_{D^{*}}$, JHEP 09 (2018) 152 [arXiv:1710.04127] [INSPIRE].

[35] D. Ghosh, M. Nardecchia and S.A. Renner, Hint of lepton flavour non-universality in B meson decays, JHEP 12 (2014) 131 [arXiv:1408.4097] [INSPIRE].

[36] S.L. Glashow, D. Guadagnoli and K. Lane, Lepton flavor violation in B decays?, Phys. Rev. Lett. 114 (2015) 091801 [arXiv: 1411.0565] [INSPIRE].

[37] B. Bhattacharya, A. Datta, D. London and S. Shivashankara, Simultaneous explanation of the $R_{K}$ and $R\left(D^{(*)}\right)$ puzzles, Phys. Lett. B 742 (2015) 370 [arXiv:1412.7164] [INSPIRE].

[38] M. Freytsis, Z. Ligeti and J.T. Ruderman, Flavor models for $\bar{B} \rightarrow D^{(*)} \tau \bar{\nu}$, Phys. Rev. D 92 (2015) 054018 [arXiv: 1506.08896] [inSPIRE].

[39] M. Ciuchini et al., On flavourful easter eggs for new physics hunger and lepton flavour universality violation, Eur. Phys. J. C 77 (2017) 688 [arXiv:1704.05447] [INSPIRE].

[40] W. Altmannshofer, S. Gori, M. Pospelov and I. Yavin, Quark flavor transitions in $L_{\mu}-L_{\tau}$ models, Phys. Rev. D 89 (2014) 095033 [arXiv:1403.1269] [INSPIRE].

[41] A. Crivellin, G. D'Ambrosio and J. Heeck, Explaining $h \rightarrow \mu^{ \pm} \tau^{\mp}, B \rightarrow K^{*} \mu^{+} \mu^{-}$and $B \rightarrow K \mu^{+} \mu^{-} / B \rightarrow K e^{+} e^{-}$in a two-Higgs-doublet model with gauged $L_{\mu}-L_{\tau}$, Phys. Rev. Lett. 114 (2015) 151801 [arXiv:1501.00993] [INSPIRE].

[42] A. Crivellin, G. D'Ambrosio and J. Heeck, Addressing the LHC flavor anomalies with horizontal gauge symmetries, Phys. Rev. D 91 (2015) 075006 [arXiv:1503.03477] [INSPIRE].

[43] D. Aristizabal Sierra, F. Staub and A. Vicente, Shedding light on the $b \rightarrow s$ anomalies with a dark sector, Phys. Rev. D 92 (2015) 015001 [arXiv:1503.06077] [INSPIRE].

[44] A. Crivellin, L. Hofer, J. Matias, U. Nierste, S. Pokorski and J. Rosiek, Lepton-flavour violating $B$ decays in generic $Z^{\prime}$ models, Phys. Rev. D 92 (2015) 054013 [arXiv: 1504.07928] [INSPIRE]. 
[45] A. Celis, J. Fuentes-Martin, M. Jung and H. Serodio, Family nonuniversal Z' models with protected flavor-changing interactions, Phys. Rev. D 92 (2015) 015007 [arXiv:1505.03079] [INSPIRE].

[46] D. Bhatia, S. Chakraborty and A. Dighe, Neutrino mixing and $R_{K}$ anomaly in $\mathrm{U}(1)_{X}$ models: a bottom-up approach, JHEP 03 (2017) 117 [arXiv:1701.05825] [INSPIRE].

[47] J.F. Kamenik, Y. Soreq and J. Zupan, Lepton flavor universality violation without new sources of quark flavor violation, Phys. Rev. D 97 (2018) 035002 [arXiv:1704.06005] [INSPIRE].

[48] C.-H. Chen and T. Nomura, Penguin $b \rightarrow s \ell^{\prime+} \ell^{\prime-}$ and B-meson anomalies in a gauged $L_{\mu}-L_{\tau}$, Phys. Lett. B 777 (2018) 420 [arXiv:1707.03249] [INSPIRE].

[49] J.E. Camargo-Molina, A. Celis and D.A. Faroughy, Anomalies in bottom from new physics in top, Phys. Lett. B 784 (2018) 284 [arXiv:1805.04917] [InSPIRE].

[50] L. Darmé, K. Kowalska, L. Roszkowski and E.M. Sessolo, Flavor anomalies and dark matter in SUSY with an extra U(1), JHEP 10 (2018) 052 [arXiv:1806.06036] [INSPIRE].

[51] S. Baek and C. Yu, Dark matter for $b \rightarrow s \mu^{+} \mu^{-}$anomaly in a gauged U(1) $X$ model, JHEP 11 (2018) 054 [arXiv: 1806.05967] [INSPIRE].

[52] A. Biswas and A. Shaw, Reconciling dark matter, $R_{K^{(*)}}$ anomalies and $(g-2)_{\mu}$ in an $L_{\mu}-L_{\tau}$ scenario, JHEP 05 (2019) 165 [arXiv: 1903.08745] [INSPIRE].

[53] B.C. Allanach and J. Davighi, Naturalising the third family hypercharge model for neutral current B-anomalies, Eur. Phys. J. C 79 (2019) 908 [arXiv:1905.10327] [InSPIRE].

[54] G. Hiller and M. Schmaltz, $R_{K}$ and future $b \rightarrow$ sll physics beyond the Standard Model opportunities, Phys. Rev. D 90 (2014) 054014 [arXiv:1408.1627] [INSPIRE].

[55] B. Gripaios, M. Nardecchia and S.A. Renner, Composite leptoquarks and anomalies in B-meson decays, JHEP 05 (2015) 006 [arXiv:1412.1791] [INSPIRE].

[56] S. Sahoo and R. Mohanta, Scalar leptoquarks and the rare B meson decays, Phys. Rev. D 91 (2015) 094019 [arXiv: 1501.05193] [INSPIRE].

[57] I. de Medeiros Varzielas and G. Hiller, Clues for flavor from rare lepton and quark decays, JHEP 06 (2015) 072 [arXiv:1503.01084] [INSPIRE].

[58] R. Alonso, B. Grinstein and J. Martin Camalich, Lepton universality violation and lepton flavor conservation in B-meson decays, JHEP 10 (2015) 184 [arXiv: 1505.05164] [INSPIRE].

[59] M. Bauer and M. Neubert, Minimal leptoquark explanation for the $R_{D^{(*)}}, R_{K}$ and $(g-2)_{g}$ anomalies, Phys. Rev. Lett. 116 (2016) 141802 [arXiv:1511.01900] [INSPIRE].

[60] C. Hati, G. Kumar and N. Mahajan, $\bar{B} \rightarrow D^{(*)} \tau \bar{\nu}$ excesses in ALRSM constrained from B, $D$ decays and $D^{0}-\bar{D}^{0}$ mixing, JHEP 01 (2016) 117 [arXiv:1511.03290] [INSPIRE].

[61] S. Fajfer and N. Košnik, Vector leptoquark resolution of $R_{K}$ and $R_{D^{(*)}}$ puzzles, Phys. Lett. B 755 (2016) 270 [arXiv:1511.06024] [INSPIRE].

[62] D. Das, C. Hati, G. Kumar and N. Mahajan, Towards a unified explanation of $R_{D^{(*)}}, R_{K}$ and $(g-2)_{\mu}$ anomalies in a left-right model with leptoquarks, Phys. Rev. D 94 (2016) 055034 [arXiv: 1605.06313] [INSPIRE]. 
[63] D. Bečirević, S. Fajfer, N. Košnik and O. Sumensari, Leptoquark model to explain the B-physics anomalies, $R_{K}$ and $R_{D}$, Phys. Rev. D 94 (2016) 115021 [arXiv:1608.08501] [INSPIRE].

[64] S. Sahoo, R. Mohanta and A.K. Giri, Explaining the $R_{K}$ and $R_{D^{(*)}}$ anomalies with vector leptoquarks, Phys. Rev. D 95 (2017) 035027 [arXiv:1609.04367] [INSPIRE].

[65] P. Cox, A. Kusenko, O. Sumensari and T.T. Yanagida, SU(5) unification with TeV-scale leptoquarks, JHEP 03 (2017) 035 [arXiv: 1612.03923] [INSPIRE].

[66] A. Crivellin, D. Müller and T. Ota, Simultaneous explanation of $R\left(D^{(*)}\right)$ and $b \rightarrow s \mu^{+} \mu^{-}$: the last scalar leptoquarks standing, JHEP 09 (2017) 040 [arXiv:1703.09226] [INSPIRE].

[67] D. Bečirević and O. Sumensari, A leptoquark model to accommodate $R_{K}^{\exp }<R_{K}^{\mathrm{SM}}$ and $R_{K^{*}}^{\exp }<R_{K^{*}}^{\mathrm{SM}}$, JHEP 08 (2017) 104 [arXiv: 1704.05835] [INSPIRE].

[68] Y. Cai, J. Gargalionis, M.A. Schmidt and R.R. Volkas, Reconsidering the one leptoquark solution: flavor anomalies and neutrino mass, JHEP 10 (2017) 047 [arXiv:1704.05849] [INSPIRE].

[69] I. Doršner, S. Fajfer, D.A. Faroughy and N. Košnik, The role of the $S_{3}$ GUT leptoquark in flavor universality and collider searches, arXiv:1706.07779 [INSPIRE].

[70] A. Greljo and B.A. Stefanek, Third family quark-lepton unification at the TeV scale, Phys. Lett. B 782 (2018) 131 [arXiv:1802.04274] [INSPIRE].

[71] S. Sahoo and R. Mohanta, Impact of vector leptoquark on $\bar{B} \rightarrow \bar{K}^{*} l^{+} l^{-}$anomalies, J. Phys. G 45 (2018) 085003 [arXiv:1806.01048] [INSPIRE].

[72] D. Bečirević, I. Doršner, S. Fajfer, N. Košnik, D.A. Faroughy and O. Sumensari, Scalar leptoquarks from grand unified theories to accommodate the B-physics anomalies, Phys. Rev. D 98 (2018) 055003 [arXiv:1806.05689] [INSPIRE].

[73] C. Hati, G. Kumar, J. Orloff and A.M. Teixeira, Reconciling B-meson decay anomalies with neutrino masses, dark matter and constraints from flavour violation, JHEP 11 (2018) 011 [arXiv:1806.10146] [INSPIRE].

[74] I. de Medeiros Varzielas and S.F. King, $R_{K^{(*)}}$ with leptoquarks and the origin of Yukawa couplings, JHEP 11 (2018) 100 [arXiv:1807.06023] [INSPIRE].

[75] J. Aebischer, A. Crivellin and C. Greub, QCD improved matching for semileptonic B decays with leptoquarks, Phys. Rev. D 99 (2019) 055002 [arXiv: 1811.08907] [INSPIRE].

[76] I. De Medeiros Varzielas and S.F. King, Origin of Yukawa couplings for Higgs bosons and leptoquarks, Phys. Rev. D 99 (2019) 095029 [arXiv:1902.09266] [INSPIRE].

[77] H. Yan, Y.-D. Yang and X.-B. Yuan, Phenomenology of $b \rightarrow c \tau \bar{\nu}$ decays in a scalar leptoquark model, Chin. Phys. C 43 (2019) 083105 [arXiv:1905.01795] [InSPIRE].

[78] I. Bigaran, J. Gargalionis and R.R. Volkas, A near-minimal leptoquark model for reconciling flavour anomalies and generating radiative neutrino masses, JHEP 10 (2019) 106 [arXiv: 1906.01870] [INSPIRE].

[79] O. Popov, M.A. Schmidt and G. White, $R_{2}$ as a single leptoquark solution to $R_{D^{(*)}}$ and $R_{K^{(*)}}$, Phys. Rev. D 100 (2019) 035028 [arXiv: 1905.06339] [InSPIRE].

[80] N.G. Deshpande and X.-G. He, Consequences of R-parity violating interactions for anomalies in $\bar{B} \rightarrow D^{(*)} \tau \bar{\nu}$ and $b \rightarrow s \mu^{+} \mu^{-}$, Eur. Phys. J. C 77 (2017) 134 [arXiv: 1608.04817] [INSPIRE]. 
[81] W. Altmannshofer, P.S. Bhupal Dev and A. Soni, $R_{D^{(*)}}$ anomaly: a possible hint for natural supersymmetry with R-parity violation, Phys. Rev. D 96 (2017) 095010 [arXiv: 1704.06659] [INSPIRE].

[82] D. Das, C. Hati, G. Kumar and N. Mahajan, Scrutinizing R-parity violating interactions in light of $R_{K^{(*)}}$ data, Phys. Rev. D 96 (2017) 095033 [arXiv: 1705.09188] [InSPIRE].

[83] K. Earl and T. Grégoire, Contributions to $b \rightarrow$ sll anomalies from R-parity violating interactions, JHEP 08 (2018) 201 [arXiv: 1806.01343] [INSPIRE].

[84] S. Trifinopoulos, Revisiting R-parity violating interactions as an explanation of the B-physics anomalies, Eur. Phys. J. C 78 (2018) 803 [arXiv: 1807.01638] [INSPIRE].

[85] S. Trifinopoulos, B-physics anomalies: the bridge between R-parity violating supersymmetry and flavoured dark matter, arXiv:1904.12940 [INSPIRE].

[86] A. Greljo, G. Isidori and D. Marzocca, On the breaking of lepton flavor universality in $B$ decays, JHEP 07 (2015) 142 [arXiv:1506.01705] [INSPIRE].

[87] P. Arnan, D. Bečirević, F. Mescia and O. Sumensari, Two Higgs doublet models and $b \rightarrow s$ exclusive decays, Eur. Phys. J. C 77 (2017) 796 [arXiv:1703.03426] [INSPIRE].

[88] L.-S. Geng, B. Grinstein, S. Jäger, J. Martin Camalich, X.-L. Ren and R.-X. Shi, Towards the discovery of new physics with lepton-universality ratios of $b \rightarrow$ sll decays, Phys. Rev. D 96 (2017) 093006 [arXiv: 1704.05446] [INSPIRE].

[89] D. Choudhury, A. Kundu, R. Mandal and R. Sinha, Minimal unified resolution to $R_{K^{(*)}}$ and $R\left(D^{(*)}\right)$ anomalies with lepton mixing, Phys. Rev. Lett. 119 (2017) 151801 [arXiv: 1706.08437] [INSPIRE].

[90] D. Choudhury, A. Kundu, R. Mandal and R. Sinha, $R_{K^{(*)}}$ and $R\left(D^{(*)}\right)$ anomalies resolved with lepton mixing, Nucl. Phys. B 933 (2018) 433 [arXiv:1712.01593] [InSPIRE].

[91] B. Grinstein, S. Pokorski and G.G. Ross, Lepton non-universality in B decays and fermion mass structure, JHEP 12 (2018) 079 [arXiv:1809.01766] [INSPIRE].

[92] D.G. Cerdeño, A. Cheek, P. Martín-Ramiro and J.M. Moreno, $B$ anomalies and dark matter: a complex connection, Eur. Phys. J. C 79 (2019) 517 [arXiv:1902.01789] [INSPIRE].

[93] S. Bhattacharya, A. Biswas, Z. Calcuttawala and S.K. Patra, An in-depth analysis of $b \rightarrow c(s)$ semileptonic observables with possible $\mu-\tau$ mixing, arXiv:1902.02796 [INSPIRE].

[94] A. Crivellin, D. Müller and C. Wiegand, $b \rightarrow s \ell^{+} \ell^{-}$transitions in two-Higgs-doublet models, JHEP 06 (2019) 119 [arXiv:1903.10440] [INSPIRE].

[95] P. Arnan, A. Crivellin, M. Fedele and F. Mescia, Generic loop effects of new scalars and fermions in $b \rightarrow s \ell^{+} \ell^{-}$and a vector-like $4^{\text {th }}$ generation, JHEP 06 (2019) 118 [arXiv: 1904.05890] [INSPIRE].

[96] N. Assad, B. Fornal and B. Grinstein, Baryon number and lepton universality violation in leptoquark and diquark models, Phys. Lett. B 777 (2018) 324 [arXiv:1708.06350] [INSPIRE].

[97] D. Buttazzo, A. Greljo, G. Isidori and D. Marzocca, B-physics anomalies: a guide to combined explanations, JHEP 11 (2017) 044 [arXiv:1706. 07808] [INSPIRE].

[98] L. Calibbi, A. Crivellin and T. Li, Model of vector leptoquarks in view of the B-physics anomalies, Phys. Rev. D 98 (2018) 115002 [arXiv:1709.00692] [INSPIRE]. 
[99] M. Bordone, C. Cornella, J. Fuentes-Martin and G. Isidori, A three-site gauge model for flavor hierarchies and flavor anomalies, Phys. Lett. B 779 (2018) 317 [arXiv:1712.01368] [INSPIRE].

[100] M. Blanke and A. Crivellin, B meson anomalies in a Pati-Salam model within the Randall-Sundrum background, Phys. Rev. Lett. 121 (2018) 011801 [arXiv:1801.07256] [INSPIRE].

[101] M. Bordone, C. Cornella, J. Fuentes-Martín and G. Isidori, Low-energy signatures of the $\mathrm{PS}^{3}$ model: from B-physics anomalies to LFV, JHEP 10 (2018) 148 [arXiv:1805.09328] [INSPIRE].

[102] J. Kumar, D. London and R. Watanabe, Combined explanations of the $b \rightarrow s \mu^{+} \mu^{-}$and $b \rightarrow c \tau^{-} \bar{\nu}$ anomalies: a general model analysis, Phys. Rev. D 99 (2019) 015007 [arXiv: 1806.07403] [INSPIRE].

[103] A. Angelescu, D. Bečirević, D.A. Faroughy and O. Sumensari, Closing the window on single leptoquark solutions to the B-physics anomalies, JHEP 10 (2018) 183 [arXiv:1808.08179] [INSPIRE].

[104] S. Balaji, R. Foot and M.A. Schmidt, Chiral SU(4) explanation of the $b \rightarrow s$ anomalies, Phys. Rev. D 99 (2019) 015029 [arXiv: 1809.07562] [INSPIRE].

[105] B. Fornal, S.A. Gadam and B. Grinstein, Left-right SU(4) vector leptoquark model for flavor anomalies, Phys. Rev. D 99 (2019) 055025 [arXiv:1812.01603] [INSPIRE].

[106] M.J. Baker, J. Fuentes-Martín, G. Isidori and M. König, High-p $p_{T}$ signatures in vector-leptoquark models, Eur. Phys. J. C 79 (2019) 334 [arXiv:1901.10480] [InSPIRE].

[107] C. Cornella, J. Fuentes-Martin and G. Isidori, Revisiting the vector leptoquark explanation of the B-physics anomalies, JHEP 07 (2019) 168 [arXiv:1903.11517] [INSPIRE].

[108] L. Da Rold and F. Lamagna, A vector leptoquark for the B-physics anomalies from a composite GUT, arXiv:1906.11666 [INSPIRE].

[109] R. Barbieri, C.W. Murphy and F. Senia, B-decay anomalies in a composite leptoquark model, Eur. Phys. J. C 77 (2017) 8 [arXiv:1611.04930] [INSPIRE].

[110] P.Q. Hung, A.J. Buras and J.D. Bjorken, Petite unification of quarks and leptons, Phys. Rev. D 25 (1982) 805 [inSPIRE].

[111] G. Valencia and S. Willenbrock, Quark-lepton unification and rare meson decays, Phys. Rev. D 50 (1994) 6843 [hep-ph/9409201] [INSPIRE].

[112] A.D. Smirnov, Mass limits for scalar and gauge leptoquarks from $K_{L}^{0} \rightarrow e^{\mp} \mu^{ \pm}, B^{0} \rightarrow e^{\mp} \tau^{ \pm}$ decays, Mod. Phys. Lett. A 22 (2007) 2353 [arXiv:0705.0308] [INSPIRE].

[113] M. Carpentier and S. Davidson, Constraints on two-lepton, two quark operators, Eur. Phys. J. C 70 (2010) 1071 [arXiv: 1008.0280] [InSPIRE].

[114] A.V. Kuznetsov, N.V. Mikheev and A.V. Serghienko, The third type of fermion mixing in the lepton and quark interactions with leptoquarks, Int. J. Mod. Phys. A 27 (2012) 1250062 [arXiv:1203.0196] [INSPIRE].

[115] A.D. Smirnov, Vector leptoquark mass limits and branching ratios of $K_{L}^{0}, B^{0}, B_{s} \rightarrow l_{i}^{+} l_{j}^{-}$ decays with account of fermion mixing in leptoquark currents, Mod. Phys. Lett. A 33 (2018) 1850019 [arXiv: 1801.02895$]$ [INSPIRE]. 
[116] F. Feruglio, P. Paradisi and A. Pattori, On the importance of electroweak corrections for B anomalies, JHEP 09 (2017) 061 [arXiv: 1705. 00929] [INSPIRE].

[117] Z.-Z. Xing, Correlation between the charged current interactions of light and heavy Majorana neutrinos, Phys. Lett. B 660 (2008) 515 [arXiv:0709.2220] [INSPIRE].

[118] M. Blennow, P. Coloma, E. Fernandez-Martinez, J. Hernandez-Garcia and J. Lopez-Pavon, Non-unitarity, sterile neutrinos and non-standard neutrino interactions, JHEP 04 (2017) 153 [arXiv: 1609.08637] [INSPIRE].

[119] E. Fernandez-Martinez, J. Hernandez-Garcia and J. Lopez-Pavon, Global constraints on heavy neutrino mixing, JHEP 08 (2016) 033 [arXiv: 1605.08774] [INSPIRE].

[120] F.J. Escrihuela, D.V. Forero, O.G. Miranda, M. Tortola and J.W.F. Valle, On the description of nonunitary neutrino mixing, Phys. Rev. D 92 (2015) 053009 [Erratum ibid. D 93 (2016) 119905] [arXiv: 1503.08879] [INSPIRE].

[121] J. Aebischer, J. Kumar and D.M. Straub, Wilson: a Python package for the running and matching of Wilson coefficients above and below the electroweak scale, Eur. Phys. J. C 78 (2018) 1026 [arXiv:1804.05033] [INSPIRE].

[122] D.M. Straub, flavio: a Python package for flavour and precision phenomenology in the Standard Model and beyond, arXiv:1810.08132 [INSPIRE].

[123] S. Jäger and J. Martin Camalich, On B $\rightarrow V \ell \ell$ at small dilepton invariant mass, power corrections and new physics, JHEP 05 (2013) 043 [arXiv:1212.2263] [INSPIRE].

[124] S. Jäger and J. Martin Camalich, Reassessing the discovery potential of the $B \rightarrow K^{*} \ell^{+} \ell^{-}$ decays in the large-recoil region: SM challenges and BSM opportunities, Phys. Rev. D 93 (2016) 014028 [arXiv: 1412.3183] [INSPIRE].

[125] M. Ciuchini et al., $B \rightarrow K^{*} \ell^{+} \ell^{-}$decays at large recoil in the Standard Model: a theoretical reappraisal, JHEP 06 (2016) 116 [arXiv:1512.07157] [INSPIRE].

[126] M. Ciuchini et al., $B \rightarrow K^{*} \ell^{+} \ell^{-}$in the Standard Model: elaborations and interpretations, PoS (ICHEP2016) 584 (2016) [arXiv: 1611.04338] [INSPIRE].

[127] I. Doršner, S. Fajfer, A. Greljo, J.F. Kamenik and N. Košnik, Physics of leptoquarks in precision experiments and at particle colliders, Phys. Rept. 641 (2016) 1 [arXiv: 1603. 04993] [INSPIRE].

[128] A. Crivellin, C. Greub, D. Müller and F. Saturnino, Importance of loop effects in explaining the accumulated evidence for new physics in $B$ decays with a vector leptoquark, Phys. Rev. Lett. 122 (2019) 011805 [arXiv: 1807.02068] [INSPIRE].

[129] A.J. Buras, D. Buttazzo, J. Girrbach-Noe and R. Knegjens, $K^{+} \rightarrow \pi^{+} \nu \bar{\nu}$ and $K_{L} \rightarrow \pi^{0} \nu \bar{\nu}$ in the Standard Model: status and perspectives, JHEP 11 (2015) 033 [arXiv:1503.02693] [INSPIRE].

[130] E949 collaboration, New measurement of the $K^{+} \rightarrow \pi^{+} \nu \bar{\nu}$ branching ratio, Phys. Rev. Lett. 101 (2008) 191802 [arXiv:0808.2459] [INSPIRE].

[131] NA62 collaboration, $K^{+} \rightarrow \pi^{+} \nu \bar{\nu}$ : first NA62 results, contribution to the $53^{\text {rd }}$ Rencontres de Moriond on Electroweak Interactions and Unified Theories (Moriond EW 2018), La Thuile, Italy, 10-17 March 2018.

[132] E391A collaboration, Experimental study of the decay $K_{L}^{0} \rightarrow \pi^{0} \nu \bar{\nu}$, Phys. Rev. D 81 (2010) 072004 [arXiv: 0911.4789] [INSPIRE]. 


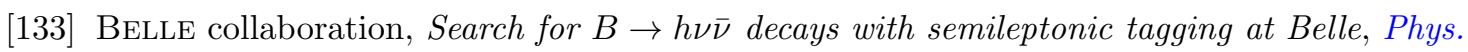
Rev. D 96 (2017) 091101 [Addendum ibid. D 97 (2018) 099902] [arXiv:1702.03224] [INSPIRE].

[134] J. Charles et al., Current status of the Standard Model CKM fit and constraints on $\Delta F=2$ new physics, Phys. Rev. D 91 (2015) 073007 [arXiv:1501.05013] [INSPIRE].

[135] J. Brod and M. Gorbahn, Next-to-next-to-leading-order charm-quark contribution to the CP-violation parameter $\epsilon_{K}$ and $\Delta M_{K}$, Phys. Rev. Lett. 108 (2012) 121801 [arXiv:1108.2036] [INSPIRE].

[136] A.J. Buras, J. Girrbach, D. Guadagnoli and G. Isidori, On the Standard Model prediction for $B R\left(B_{s, d} \rightarrow \mu^{+} \mu^{-}\right)$, Eur. Phys. J. C 72 (2012) 2172 [arXiv:1208.0934] [INSPIRE].

[137] B. Capdevila, A. Crivellin, S. Descotes-Genon, L. Hofer and J. Matias, Searching for new physics with $b \rightarrow s \tau^{+} \tau^{-}$processes, Phys. Rev. Lett. 120 (2018) 181802 [arXiv:1712.01919] [INSPIRE].

[138] MEG collaboration, Search for the lepton flavour violating decay $\mu^{+} \rightarrow e^{+} \gamma$ with the full dataset of the MEG experiment, Eur. Phys. J. C 76 (2016) 434 [arXiv:1605.05081] [INSPIRE].

[139] MEG II collaboration, The design of the MEG II experiment, Eur. Phys. J. C 78 (2018) 380 [arXiv: 1801.04688] [INSPIRE].

[140] BABAR collaboration, Searches for lepton flavor violation in the decays $\tau^{ \pm} \rightarrow e^{ \pm} \gamma$ and $\tau^{ \pm} \rightarrow \mu^{ \pm} \gamma$, Phys. Rev. Lett. 104 (2010) 021802 [arXiv:0908.2381] [INSPIRE].

[141] BeLLE-II collaboration, The Belle II physics book, arXiv:1808.10567 [INSPIRE].

[142] SINDRUM collaboration, Search for the decay $\mu^{+} \rightarrow e^{+} e^{+} e^{-}$, Nucl. Phys. B 299 (1988) 1 [INSPIRE].

[143] A. Blondel et al., Research proposal for an experiment to search for the decay $\mu \rightarrow$ eee, arXiv:1301.6113 [INSPIRE].

[144] K. Hayasaka et al., Search for lepton flavor violating $\tau$ decays into three leptons with 719 million produced $\tau^{+} \tau^{-}$pairs, Phys. Lett. B 687 (2010) 139 [arXiv:1001.3221] [INSPIRE].

[145] SINDRUM II collaboration, A search for muon to electron conversion in muonic gold, Eur. Phys. J. C 47 (2006) 337 [inSPIRE].

[146] DeEMe collaboration, Search for $\mu-e$ conversion with DeeMe experiment at J-PARC $M L F$, PoS (FPCP2015) 060 (2015) [INSPIRE].

[147] COMET collaboration, An overview of the COMET experiment and its recent progress, in Proceedings, $17^{\text {th }}$ International Workshop on Neutrino Factories and Future Neutrino Facilities (NuFact15), Rio de Janeiro, Brazil, 10-15 August 2015 [arXiv: 1512.08564] [INSPIRE].

[148] Y. Kuno, Physics prospects with muons, presentation at the Flavour Session of the CERN Council Open Symposium on the Update of the European Strategy for Particle Physics, Granada, Spain, 13-16 May 2019.

[149] Mu2E collaboration, Mu2e technical design report, arXiv:1501.05241 [INSPIRE].

[150] Mu2E collaboration, Expression of interest for evolution of the Mu2e experiment, arXiv: 1802.02599 [INSPIRE]. 
[151] CMS collaboration, Search for pair production of third-generation scalar leptoquarks and top squarks in proton-proton collisions at $\sqrt{s}=8 \mathrm{TeV}$, Phys. Lett. B 739 (2014) 229 [arXiv:1408.0806] [INSPIRE].

[152] ATLAS collaboration, Searches for scalar leptoquarks in pp collisions at $\sqrt{s}=8 \mathrm{TeV}$ with the ATLAS detector, Eur. Phys. J. C 76 (2016) 5 [arXiv:1508.04735] [INSPIRE].

[153] CMS collaboration, Search for third-generation scalar leptoquarks and heavy right-handed neutrinos in final states with two $\tau$ leptons and two jets in proton-proton collisions at $\sqrt{s}=13 \mathrm{TeV}$, JHEP 07 (2017) 121 [arXiv: 1703.03995] [INSPIRE].

[154] CMS collaboration, Search for heavy neutrinos and third-generation leptoquarks in hadronic states of two $\tau$ leptons and two jets in proton-proton collisions at $\sqrt{s}=13 \mathrm{TeV}$, JHEP 03 (2019) 170 [arXiv: 1811.00806] [INSPIRE].

[155] CMS collaboration, Constraints on models of scalar and vector leptoquarks decaying to a quark and a neutrino at $\sqrt{s}=13$ TeV, Phys. Rev. D 98 (2018) 032005 [arXiv:1805.10228] [INSPIRE].

[156] A. Cerri et al., Opportunities in flavour physics at the HL-LHC and HE-LHC, arXiv: 1812.07638 [INSPIRE].

[157] Working Group 3 collaboration, Beyond the Standard Model physics at the HL-LHC and HE-LHC, arXiv: 1812.07831 [INSPIRE].

[158] G. Buchalla, A.J. Buras and M.E. Lautenbacher, Weak decays beyond leading logarithms, Rev. Mod. Phys. 68 (1996) 1125 [hep-ph/9512380] [INSPIRE].

[159] C. Bobeth, M. Misiak and J. Urban, Photonic penguins at two loops and $m_{t}$ dependence of $B R\left[B \rightarrow X_{s} l^{+} l^{-}\right]$, Nucl. Phys. B 574 (2000) 291 [hep-ph/9910220] [INSPIRE].

[160] A. Ali, E. Lunghi, C. Greub and G. Hiller, Improved model independent analysis of semileptonic and radiative rare B decays, Phys. Rev. D 66 (2002) 034002 [hep-ph/0112300] [INSPIRE].

[161] G. Hiller and F. Krüger, More model-independent analysis of $b \rightarrow s$ processes, Phys. Rev. D 69 (2004) 074020 [hep-ph/0310219] [inSPIRE].

[162] C. Bobeth, G. Hiller and G. Piranishvili, Angular distributions of $\bar{B} \rightarrow \bar{K} \ell^{+} \ell^{-}$decays, JHEP 12 (2007) 040 [arXiv:0709.4174] [INSPIRE].

[163] C. Bobeth, G. Hiller and D. van Dyk, The benefits of $\bar{B} \rightarrow \bar{K}^{*} l^{+} l^{-}$decays at low recoil, JHEP 07 (2010) 098 [arXiv: 1006.5013] [InSPIRE].

[164] D. Bečirević, O. Sumensari and R. Zukanovich Funchal, Lepton flavor violation in exclusive $b \rightarrow s$ decays, Eur. Phys. J. C 76 (2016) 134 [arXiv:1602.00881] [InSPIRE].

[165] A. Khodjamirian, T. Mannel, A.A. Pivovarov and Y.-M. Wang, Charm-loop effect in $B \rightarrow K^{(*)} \ell^{+} \ell^{-}$and $B \rightarrow K^{*} \gamma$, JHEP 09 (2010) 089 [arXiv: 1006.4945] [INSPIRE].

[166] A.J. Buras, J. Girrbach-Noe, C. Niehoff and D.M. Straub, $B \rightarrow K^{(*)} \nu \bar{\nu}$ decays in the Standard Model and beyond, JHEP 02 (2015) 184 [arXiv:1409.4557] [INSPIRE].

[167] C. Bobeth and A.J. Buras, Leptoquarks meet $\epsilon^{\prime} / \epsilon$ and rare Kaon processes, JHEP 02 (2018) 101 [arXiv: 1712.01295] [INSPIRE].

[168] M. Bordone, D. Buttazzo, G. Isidori and J. Monnard, Probing lepton flavour universality with $K \rightarrow \pi \nu \bar{\nu}$ decays, Eur. Phys. J. C 77 (2017) 618 [arXiv:1705.10729] [InSPIRE]. 
[169] A.J. Buras, T. Ewerth, S. Jager and J. Rosiek, $K^{+} \rightarrow \pi^{+} \nu \bar{\nu}$ and $K_{L} \rightarrow \pi^{0} \nu \bar{\nu}$ decays in the general MSSM, Nucl. Phys. B 714 (2005) 103 [hep-ph/0408142] [INSPIRE].

[170] L. Lavoura, General formulae for $f_{1} \rightarrow f_{2} \gamma$, Eur. Phys. J. C 29 (2003) 191 [hep-ph/0302221] [INSPIRE].

[171] Y. Okada, K.-I. Okumura and Y. Shimizu, $\mu \rightarrow e \gamma$ and $\mu \rightarrow 3 e$ processes with polarized muons and supersymmetric grand unified theories, Phys. Rev. D 61 (2000) 094001 [hep-ph/9906446] [INSPIRE].

[172] Y. Kuno and Y. Okada, Muon decay and physics beyond the Standard Model, Rev. Mod. Phys. 73 (2001) 151 [hep-ph/9909265] [INSPIRE].

[173] E. Gabrielli, Model independent constraints on leptoquarks from rare muon and tau lepton processes, Phys. Rev. D 62 (2000) 055009 [hep-ph/9911539] [INSPIRE].

[174] R. Kitano, M. Koike and Y. Okada, Detailed calculation of lepton flavor violating muon electron conversion rate for various nuclei, Phys. Rev. D 66 (2002) 096002 [Erratum ibid. D 76 (2007) 059902] [hep-ph/0203110] [INSPIRE].

[175] T.S. Kosmas, S. Kovalenko and I. Schmidt, Nuclear $\mu^{-}-e^{-}$conversion in strange quark sea, Phys. Lett. B 511 (2001) 203 [hep-ph/0102101] [INSPIRE].

[176] Z. Poh and S. Raby, Vectorlike leptons: muon $g-2$ anomaly, lepton flavor violation, Higgs boson decays and lepton nonuniversality, Phys. Rev. D 96 (2017) 015032 [arXiv: 1705. 07007] [INSPIRE].

[177] R. Dermisek and A. Raval, Explanation of the muon $g-2$ anomaly with vectorlike leptons and its implications for Higgs decays, Phys. Rev. D 88 (2013) 013017 [arXiv:1305.3522] [INSPIRE].

[178] A. Abada, A.M. Teixeira, A. Vicente and C. Weiland, Sterile neutrinos in leptonic and semileptonic decays, JHEP 02 (2014) 091 [arXiv:1311.2830] [INSPIRE].

[179] A. Atre, T. Han, S. Pascoli and B. Zhang, The search for heavy Majorana neutrinos, JHEP 05 (2009) 030 [arXiv: 0901.3589] [INSPIRE].

[180] A. Abada, V. De Romeri, M. Lucente, A.M. Teixeira and T. Toma, Effective Majorana mass matrix from $\tau$ and pseudoscalar meson lepton number violating decays, JHEP 02 (2018) 169 [arXiv: 1712.03984] [INSPIRE].

[181] A. Abada, C. Hati, X. Marcano and A.M. Teixeira, Interference effects in LNV and LFV semileptonic decays: the Majorana hypothesis, JHEP 09 (2019) 017 [arXiv: 1904.05367] [INSPIRE].

[182] S. Bray, J.S. Lee and A. Pilaftsis, Resonant CP-violation due to heavy neutrinos at the LHC, Nucl. Phys. B 786 (2007) 95 [hep-ph/0702294] [INSPIRE].

[183] P.S. Bhupal Dev, R.N. Mohapatra and Y. Zhang, CP violating effects in heavy neutrino oscillations: implications for colliders and leptogenesis, arXiv:1904.04787 [INSPIRE].

[184] J. Aebischer, J. Kumar, P. Stangl and D.M. Straub, A global likelihood for precision constraints and flavour anomalies, Eur. Phys. J. C 79 (2019) 509 [arXiv:1810.07698] [INSPIRE]. 